\title{
Characterization of the convolution operators on quasianalytic classes of Beurling type that admit a continuous linear right inverse
}

\author{
by \\ José Bonet (Valencia) and Reinhold Meise (Düsseldorf)
}

\begin{abstract}
Extending previous work by Meise and Vogt, we characterize those convolution operators, defined on the space $\mathcal{E}_{(\omega)}(\mathbb{R})$ of $(\omega)$-quasianalytic functions of Beurling type of one variable, which admit a continuous linear right inverse. Also, we characterize those $(\omega)$-ultradifferential operators which admit a continuous linear right inverse on $\mathcal{E}_{(\omega)}[a, b]$ for each compact interval $[a, b]$ and we show that this property is in fact weaker than the existence of a continuous linear right inverse on $\mathcal{E}_{(\omega)}(\mathbb{R})$.
\end{abstract}

1. Introduction. For a weight function $\omega$ let $\mathcal{E}_{(\omega)}(\mathbb{R})$ denote the space of all $(\omega)$-ultradifferentiable functions of Beurling type on $\mathbb{R}$. Then each $\mu \in \mathcal{E}_{(\omega)}^{\prime}(\mathbb{R})$ induces a convolution operator $T_{\mu}: \mathcal{E}_{(\omega)}(\mathbb{R}) \rightarrow \mathcal{E}_{(\omega)}(\mathbb{R})$. If $\omega$ is non-quasianalytic, i.e., if $\mathcal{E}_{(\omega)}(\mathbb{R})$ admits non-trivial functions with compact support, then Meise and Vogt [21] characterized by various equivalences those convolution operators $T_{\mu}$ on $\mathcal{E}_{(\omega)}(\mathbb{R})$ that admit a continuous linear right inverse. The arguments which they apply in their proofs use the nonquasianalyticity in an essential way, in particular through the existence of cut-off functions and fundamental solutions.

In the present paper we first show that for each quasianalytic weight function $\omega$ which satisfies condition $\left(\alpha_{1}\right)$, a convolution operator $T_{\mu}$ on $\mathcal{E}_{(\omega)}(\mathbb{R})$ admits a continuous linear right inverse only if its Fourier-Laplace transform $\widehat{\mu}$ is $(\omega)$-slowly decreasing and satisfies $|\operatorname{Im} a|=O(\omega(a))$ for $a \in V(\widehat{\mu})$ as $|a|$ tends to infinity. Conversely, if these two conditions hold and if $\omega$ is a (DN)-weight function, then $T_{\mu}$ admits a continuous linear right inverse on $\mathcal{E}_{(\omega)}(\mathbb{R})$, even without the assumption that $\omega$ satisfies condition $\left(\alpha_{1}\right)$. Accordingly, when $\omega$ is a quasianalytic $(\mathrm{DN})$-weight function satisfying condition $\left(\alpha_{1}\right)$, we obtain a characterization (see Theorem 3.4). To

2000 Mathematics Subject Classification: Primary 42A85, 30D60; Secondary 46E10, 47B38.

Key words and phrases: quasianalytic classes of Beurling type, convolution operators, continuous linear right inverse, Fourier-Laplace transform, property (DN). 
prove these results we use an idea of Langenbruch [13] who characterized the convolution operators on the real-analytic functions on $\mathbb{R}$ which admit a solution operator. Also, we work with the space of Fourier-Laplace transforms of the $(\omega)$-quasianalytic functionals, where we apply methods that go back to Berenstein and Taylor [2], Meise [15], and Meise and Taylor [17]. An important step in proving the necessity of the conditions given above is a recent result of Vogt [30] and Bonet and Domański [4] on topological invariants of spaces of $\{\omega\}$-ultradifferentiable functions of Roumieu type.

We also investigate $(\omega)$-ultradifferential operators $T_{\mu}$ on $\mathcal{E}_{(\omega)}(\mathbb{R})$ and on $\mathcal{E}_{(\omega)}[a, b]$ for compact intervals $[a, b]$. It turns out that the surjectivity of such an operator on $\mathcal{E}_{(\omega)}(\mathbb{R})$ already implies the existence of a continuous linear right inverse for $T_{\mu}$, restricted to $\mathcal{E}_{(\omega)}[a, b]$. As a consequence we deduce that an analogue of a result of Domański and Vogt [9, Theorem 4.7] in the realanalytic case also holds for the class $\mathcal{E}_{(\omega)}$, provided that $\omega$ is a quasianalytic $(\mathrm{DN})$-weight function which satisfies condition $\left(\alpha_{1}\right)$. More precisely, we show that each $(\omega)$-ultradifferential operator $T_{\mu}$ which is surjective on $\mathcal{E}_{(\omega)}(\mathbb{R})$ admits a continuous linear right inverse on $\mathcal{E}_{(\omega)}(\mathbb{R})$ if and only if for each compact interval $[a, b]$ and each $f \in \mathcal{E}_{(\omega)}[a, b]$ satisfying $T_{\mu}(f)=0$ there exists $g \in \mathcal{E}_{(\omega)}(\mathbb{R})$ satisfying $T_{\mu}(g)=0$ and $f=\left.g\right|_{[a, b]}$.

The authors thank P. Domański and D. Vogt for a discussion which drew their attention to the paper [9]. We also thank the referee for the careful reading of our manuscript.

Acknowledgements. The present research was partially supported by FEDER and MEC Project MTM2004-02262 and the research net MTM200626627-E. This article was completed during a stay of R. Meise at the Instituto de Matemática Pura y Aplicada IMPA-UPV of the Universidad Politécnica de Valencia in February/March 2007 under the support of the grant AINV/2007/050 from Generalitat Valenciana.

2. Preliminaries. In this section we introduce the notation that will be used throughout the entire paper.

2.1. Weight functions. A function $\omega: \mathbb{R} \rightarrow[0, \infty[$ is called a weight function if it is continuous, even, increasing on $[0, \infty[$, satisfies $\omega(0)=0$, and also the following conditions:

$(\alpha)$ There exists $K \geq 1$ such that $\omega(2 t) \leq K \omega(t)+K$ for all $t \geq 0$.

$(\beta) \omega(t)=o(t)$ as $t$ tends to infinity.

$(\gamma) \log (t)=o(\omega(t))$ as $t$ tends to infinity.

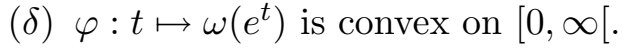


(a) If a weight function $\omega$ satisfies

$$
\int_{1}^{\infty} \frac{\omega(t)}{t^{2}} d t=\infty,
$$

then it is called a quasianalytic weight function. Otherwise it is called nonquasianalytic.

(b) If a weight function $\omega$ satisfies the condition

( $\varepsilon$ ) there exists $C>0$ such that $\int_{1}^{\infty}\left(\omega(y t) / t^{2}\right) d t \leq C \omega(y)+C$ for all $y>0$,

then $\omega$ is called a strong weight function. Obviously, each strong weight function is non-quasianalytic. The reverse implication does not hold.

(c) A weight function $\omega$ satisfies condition $\left(\alpha_{1}\right)$ if

$$
\sup _{\lambda \geq 1} \limsup _{t \rightarrow \infty} \frac{\omega(\lambda t)}{\lambda \omega(t)}<\infty .
$$

This condition was introduced by Petzsche and Vogt [26] and is equivalent to the existence of $C_{1}>0$ such that for each $W \geq 1$ there exists $C_{2}>0$ such that

$$
\omega(W t+W) \leq W C_{1} \omega(t)+C_{2}, t \geq 0 .
$$

(d) The radial extension $\widetilde{\omega}$ of a weight function $\omega$ is defined as

$$
\widetilde{\omega}: \mathbb{C}^{n} \rightarrow[0, \infty[, \quad \widetilde{\omega}(z):=\omega(|z|) .
$$

It will also be denoted by $\omega$, by abuse of notation.

(e) The Young conjugate of the function $\varphi=\varphi_{\omega}$, which appears in $(\delta)$, is defined as

$$
\varphi^{*}(x):=\sup \{x y-\varphi(y): y>0\}, \quad x \geq 0 .
$$

2.2. EXAmple. The following are easily seen to be weight functions:

(1) $\omega(t):=|t|(\log (e+|t|))^{-\alpha}, \alpha>0$.

(2) $\omega(t):=|t|^{\alpha}, 0<\alpha<1$.

(3) $\omega(t)=(\max (0, \log t))^{s}, s>1$.

2.3. Ultradifferentiable functions defined by weight functions. Let $\omega$ be a given weight function, let $K$ be a compact and $G$ be an open subset of $\mathbb{R}^{N}$, and denote by $C^{\infty}(K)$ the space of all $C^{\infty}$-Whitney jets on $K$.

(a) The space $\mathcal{E}_{(\omega)}(G)$ of $(\omega)$-ultradifferentiable functions of Beurling type on $G$ is defined as

$$
\begin{aligned}
& \mathcal{E}_{(\omega)}(G):=\left\{f \in C^{\infty}(G): \text { for each } K \subset G \text { compact and } m \in \mathbb{N},\right. \\
&\left.p_{K, m}(f):=\sup _{x \in K} \sup _{\alpha \in \mathbb{N}_{0}^{N}}\left|f^{(\alpha)}(x)\right| \exp \left(-m \varphi^{*}(|\alpha| / m)\right)<\infty\right\} .
\end{aligned}
$$

It is easy to check that $\mathcal{E}_{(\omega)}(G)$ is a Fréchet space if we endow it with the locally convex topology given by the seminorms $p_{K, m}$. We also define the 
space

$$
\mathcal{E}_{(\omega)}(K):=\left\{f \in C^{\infty}(K): p_{K, m}(f)<\infty \forall m \in \mathbb{N}\right\} .
$$

(b) For $m \in \mathbb{N}$ let

$$
\begin{aligned}
\mathcal{E}_{\{\omega\}}^{m}(K):= & \left\{f \in C^{\infty}(K):\right. \\
& \left.\|f\|_{K, m}:=\sup _{x \in K} \sup _{\alpha \in \mathbb{N}_{0}^{N}}\left|f^{(\alpha)}(x)\right| \exp \left(-\frac{1}{m} \varphi^{*}(m|\alpha|)\right)<\infty\right\}
\end{aligned}
$$

and define the space $\mathcal{E}_{\{\omega\}}(G)$ of $\{\omega\}$-ultradifferentiable functions of Roumieu type on $G$ as

$$
\begin{aligned}
\mathcal{E}_{\{\omega\}}(G):=\left\{f \in C^{\infty}(G): \text { for each } K \subset G\right. \text { compact } \\
\text { there is } \left.m \in \mathbb{N} \text { so that }\|f\|_{K, m}<\infty\right\} .
\end{aligned}
$$

It is endowed with the topology given by the representation

$$
\mathcal{E}_{\{\omega\}}(G)=\operatorname{proj}_{\leftarrow K} \operatorname{ind}_{m \rightarrow} \mathcal{E}_{\{\omega\}}^{m}(K),
$$

where $K$ runs over all compact subsets of $G$.

Note that $\mathcal{E}_{\{\omega\}}(G)$ is a countable projective limit of (DFN)-spaces, which is ultrabornological, reflexive and complete. This follows from Rösner [27, Satz 3.25] and Vogt [30, Theorem 3.4].

If a statement holds in the Beurling and the Roumieu case then we will use the notation $\mathcal{E}_{*}(G)$. It means that in all cases $*$ can be replaced either by $(\omega)$ or by $\{\omega\}$.

2.4. Definition. Let $\omega$ be a weight function and $G$ an open convex set in $\mathbb{R}^{N}$.

(a) We define

$$
A_{(\omega)}:=\left\{f \in H\left(\mathbb{C}^{N}\right): \exists n \in \mathbb{N}:\|f\|_{n}:=\sup _{z \in \mathbb{C}}|f(z)| \exp (-n \omega(z))<\infty\right\} .
$$

Endowed with its natural (LB)-topology, $A_{(\omega)}$ is a $(\mathrm{DFN})$-space.

(b) For each compact set $K$ in $G$, the support functional of $K$ is

$$
h_{K}: \mathbb{R}^{N} \rightarrow \mathbb{R}, \quad h_{K}(x):=\sup \{\langle x, y\rangle: y \in K\} .
$$

(c) For $K$ as in (b) and $\lambda>0$ let

$$
\begin{aligned}
A(K, \lambda):=\left\{f \in H\left(\mathbb{C}^{N}\right):\right. & \\
& \left.\|f\|_{K, \lambda}:=\sup _{z \in \mathbb{C}^{N}}|f(z)| \exp \left(-h_{K}(\operatorname{Im} z)-\lambda \omega(|z|)\right)<\infty\right\}
\end{aligned}
$$

and define

$$
\begin{aligned}
& A_{(\omega)}\left(\mathbb{C}^{N}, G\right):=\operatorname{ind}_{K, n \rightarrow} A(K, n),
\end{aligned}
$$

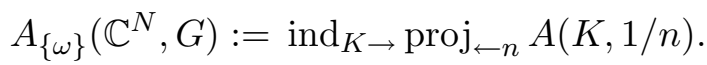


It is easy to check that $A(K, \lambda)$ is a Banach space, that $A_{(\omega)}\left(\mathbb{C}^{N}, G\right)$ is an (LB)-space, and that $A_{\{\omega\}}\left(\mathbb{C}^{N}, G\right)$ is an (LF)-space.

2.5. The Fourier-Laplace transform. Let $\omega$ be a weight function and let $G$ be an open convex set in $\mathbb{R}^{N}$. For each $u \in \mathcal{E}_{*}(G)^{\prime}$ it is easy to check that

$$
\widehat{u}: \mathbb{C}^{N} \rightarrow \mathbb{C}, \quad \widehat{u}(z):=u_{x}\left(e^{-i\langle x, z\rangle}\right),
$$

is an entire function which belongs to $A_{*}\left(\mathbb{C}^{N}, G\right)$ and that

$$
\mathcal{F}: \mathcal{E}_{*}^{\prime}(G) \rightarrow A_{*}\left(\mathbb{C}^{N}, G\right), \quad \mathcal{F}(u):=\widehat{u},
$$

is linear and continuous.

The following result was proved for $N=1$ by Meyer [23] and for $N \geq 1$ in the Roumieu case by Rösner [27]. For a unified proof we refer to Heinrich and Meise [10, Theorems 3.6 and 3.7].

2.6. TheOREM. For each weight function $\omega$ and each convex open set $G \subset \mathbb{R}^{N}$ the Fourier-Laplace transform

$$
\mathcal{F}: \mathcal{E}_{*}^{\prime}(G) \rightarrow A_{*}\left(\mathbb{C}^{N}, G\right)
$$

is a linear topological isomorphism.

2.7. Convolution operators. For $\mu \in \mathcal{E}_{*}(\mathbb{R})^{\prime}, \mu \neq 0$, and $\varphi \in \mathcal{E}_{*}(\mathbb{R})$ we define

$$
\check{\mu}(\varphi):=\mu(\check{\varphi}), \quad \check{\varphi}(x):=\varphi(-x), \quad x \in \mathbb{R} .
$$

The convolution operator $T_{\mu}: \mathcal{E}_{*}(\mathbb{R}) \rightarrow \mathcal{E}_{*}(\mathbb{R})$ is defined by

$$
T_{\mu}(f):=\check{\mu} * f, \quad(\check{\mu} * f)(x):=\check{\mu}(f(x-\cdot)), \quad x \in \mathbb{R} .
$$

It is a well-defined, linear, continuous operator; see Meyer [23] and [24]. For $g \in A_{*}(\mathbb{C}, \mathbb{R})$ we define the multiplication operator $M_{g}: A_{*}(\mathbb{C}, \mathbb{R}) \rightarrow$ $A_{*}(\mathbb{C}, \mathbb{R})$ by $M_{g}(f)=g f$. It is well-known that for $\mu \in \mathcal{E}_{*}(\mathbb{R})$ we have $\mathcal{F} \circ T_{\mu}^{t}=M_{\widehat{\mu}} \circ \mathcal{F}$ on $\mathcal{E}_{*}^{\prime}(\mathbb{R})$.

By the work of S. Momm [25, Proposition 2 and Corollary 1] (see also [5, Proposition 2.6 and Theorem 2.7]) we have

2.8. THEOREM. For each weight function $\omega$ the following conditions are equivalent for $\mu \in \mathcal{E}_{(\omega)}^{\prime}(\mathbb{R}), \mu \neq 0$ :

(1) $T_{\mu}: \mathcal{E}_{(\omega)}(\mathbb{R}) \rightarrow \mathcal{E}_{(\omega)}(\mathbb{R})$ is surjective.

(2) The principal ideal $\widehat{\mu} A_{(\omega)}(\mathbb{C}, \mathbb{R})$ is closed in $A_{(\omega)}(\mathbb{C}, \mathbb{R})$.

(3) $\widehat{\mu}$ is $(\omega)$-slowly decreasing in the sense of Ehrenpreis, i.e., there exist $k, x_{0}>0$ such that for each $x \in \mathbb{R}$ with $|x| \geq x_{0}$ there exists $t \in \mathbb{R}$ with $|t-x| \leq k \omega(x)$ such that

$$
|\widehat{\mu}(t)| \geq \exp (-k \omega(t)) .
$$


(4) $\widehat{\mu}$ is $(\omega)$-slowly decreasing, i.e., there exists $C>0$ such that for each $x \in \mathbb{R}$ with $|x| \geq C$ there exists $\xi \in \mathbb{C}$ such that

$$
|x-\xi| \leq C \omega(x), \quad|\widehat{\mu}(\xi)| \geq \exp (-C|\operatorname{Im} \xi|-C \omega(\xi)) .
$$

(5) $\widehat{\mu}$ is slowly decreasing for $A_{(\omega)}(\mathbb{C}, \mathbb{R})$ in the sense of Berenstein and Taylor, i.e., for $p(z):=|\operatorname{Im} z|+\omega(z), z \in \mathbb{C}$, there exist $\varepsilon, C, D>0$ such that each connected component $S$ of

satisfies

$$
S(\widehat{\mu}, \varepsilon, C):=\{z \in \mathbb{C}:|\widehat{\mu}(z)|<\varepsilon \exp (-C p(z))\}
$$

$$
\sup _{z \in S} p(z) \leq D\left(1+\inf _{z \in S} p(z)\right) \text {. }
$$

(6) There exist $k \in \mathbb{N}, m \in \mathbb{N}$, and $R>0$ such that for each $z \in \mathbb{C}$ with $|z| \geq R$ there is a circle $T$ surrounding $z$ with diameter $d(T) \leq$ $|\operatorname{Im} z|+k \omega(z)$ and $|F(w)| \geq \exp (-m p(w))$ for each $w \in T$.

In the next section we will use the following definitions.

2.9. Definition. Let $\alpha=\left(\alpha_{j}\right)_{j \in \mathbb{N}}$ be an increasing, unbounded sequence in $\left[0, \infty\left[\right.\right.$. For $R \in\{0, \infty\}$ the power series spaces $\Lambda_{R}(\alpha)$ are defined as

$$
\Lambda_{R}(\alpha):=\left\{x=\left(x_{j}\right)_{j \in \mathbb{N}} \in \mathbb{C}^{\mathbb{N}}:\|x\|_{r}:=\sum_{j=1}^{\infty}\left|x_{j}\right| \exp \left(r \alpha_{j}\right)<\infty \forall r<R\right\} .
$$

$\Lambda_{\infty}(\alpha)$ is called a power series space of infinite type, while $\Lambda_{0}(\alpha)$ is said to be of finite type. Note that $\Lambda_{R}(\alpha)$ is a Fréchet-Schwartz space for each $\alpha$ and each $R$.

The following linear topological invariants are related to power series spaces of infinite type. For details we refer to Meise and Vogt [22, Section 29].

2.10. Definition. Let $E$ be a Fréchet space and let $\left(\|\cdot\|_{j}\right)_{j \in \mathbb{N}}$ be a fundamental sequence of seminorms for $E$.

(a) $E$ has property (DN) if there exists $p \in \mathbb{N}$ such that for each $k \in \mathbb{N}$ there exist $n \in \mathbb{N}$ and $C>0$ such that

$$
\|x\|_{k}^{2} \leq C\|x\|_{p}\|x\|_{n}, \quad x \in E .
$$

(b) $E$ has property $(\Omega)$ if for each $p \in \mathbb{N}$ there exists $q \in \mathbb{N}$ such that for each $k \in \mathbb{N}$ and $0<\Theta<1$ there exists $C>0$ such that

$$
\|y\|_{q}^{*} \leq C\|y\|_{p}^{* 1-\Theta}\|y\|_{k}^{* \Theta}, \quad y \in E^{\prime},
$$

where for $y \in E^{\prime}$,

$$
\|y\|_{k}^{*}:=\sup \left\{|y(x)|:\|x\|_{k} \leq 1\right\} \in \mathbb{R} \cup\{+\infty\} .
$$

Note that each subspace of a power series space of infinite type has (DN), while each quotient space has $(\Omega)$.

From Meise and Taylor [18], we recall the following definition. 
2.11. Definition. A weight function $\omega$ is called a (DN)-weight function if it satisfies:

(2.1) for each $C>1$ there exist $R_{0}>0$ and $0<\delta<1$ such that for each $R \geq R_{0}$

$$
\omega^{-1}(C R) \omega^{-1}(\delta R) \leq\left(\omega^{-1}(R)\right)^{2} .
$$

For the significance of (DN)-weight functions we refer to Meise and Taylor [18, Theorem 3.4].

2.12. ExAMPLE. Let $\omega$ be a weight function for which there exists $A>0$ such that

$$
2 \omega(t) \leq \omega(A t)+A, \quad t \geq 0 .
$$

Then $\omega$ is a (DN)-weight function by Meise and Taylor [18, Example 3.5(4)]. In particular, the following functions are quasianalytic (DN)-weight functions which also satisfy $\left(\alpha_{1}\right)$ :

(1) $\omega(t):=|t|(\log (e+|t|))^{-\alpha}, 0<\alpha \leq 1$.

(2) $\omega(t):=|t|(\log (e+\log (e+|t|)))^{-1}$.

3. The global case. In this section we characterize when a convolution operator $T_{\mu}$ admits a continuous linear right inverse on $\mathcal{E}_{(\omega)}(\mathbb{R})$. To do this we will use the following lemma.

3.1. LEMmA. Let $\omega$ be a weight function which satisfies condition $\left(\alpha_{1}\right)$. Suppose that there is a sequence $\left(a_{j}\right)_{j \in \mathbb{N}}$ of complex numbers with $0<\left|a_{j}\right|<$ $\left|a_{j+1}\right|$ for each $j \in \mathbb{N}$ and $\lim _{j \rightarrow \infty}\left|a_{j}\right|=\infty$ such that $\omega\left(a_{j}\right) \leq\left|\operatorname{Im} a_{j}\right| / j^{2}$ for each $j \in \mathbb{N}$. Then there is a weight function $\sigma$ satisfying condition $\left(\alpha_{1}\right)$ and $\omega(t)=o(\sigma(t))$ as $t \rightarrow \infty$, and there is a subsequence $\left(a_{j(k)}\right)_{k \in \mathbb{N}}$ of $\left(a_{j}\right)_{j \in \mathbb{N}}$ such that $\sigma\left(a_{j(k)}\right)=O\left(\left|\operatorname{Im} a_{j(k)}\right|\right)$ as $k \rightarrow \infty$.

Proof. CASE 1: $\left|\operatorname{Im} a_{j}\right|=o\left(\left|a_{j}\right|\right)$ as $j \rightarrow \infty$. Then we write $a_{j}=\alpha_{j}+i \beta_{j}$, $j \in \mathbb{N}$, and choose $j(1) \in \mathbb{N}$ such that $\left|\beta_{j}\right| \leq\left|\alpha_{j}\right| / 2$ and $\omega\left(\alpha_{j}\right) \geq 1$ if $j \geq j(1)$. By the properties of $\omega$, there exists $D \geq 1$ such that for all $j \geq j(1)$,

$$
\omega\left(\alpha_{j}\right) \leq \omega\left(\left|a_{j}\right|\right) \leq \omega\left(\left|\alpha_{j}\right|+\left|\beta_{j}\right|\right) \leq \omega\left(\frac{3}{2}\left|\alpha_{j}\right|\right) \leq D \omega\left(\left|\alpha_{j}\right|\right)+D \leq 2 D \omega\left(\alpha_{j}\right) .
$$

Without restriction we may assume that $\alpha_{j}>0$ for $j \geq j(1)$. Then we define $x_{1}:=0$ and $x_{2}:=\alpha_{j(1)}$. Proceeding by induction suppose that $j(n)>$ $\max (n, j(n-1))$ and $x_{n+1}:=\alpha_{j(n)}>2 x_{n}$ are already selected satisfying

$$
\omega\left(x_{n+1}\right) \geq 2^{n-i+1} \omega\left(x_{i}\right), 1 \leq i \leq n, \quad \text { and } \quad \omega(x) \leq x / n^{2} \quad \text { if } x \geq x_{n} .
$$

Since $\left(\left|\alpha_{j}\right|\right)_{j \in \mathbb{N}}$ tends to infinity and $\omega$ satisfies condition $2.1(\gamma)$ and $\lim _{t \rightarrow \infty} \omega(t) / t=0$, we can find $j(n+1)>\max (n+1, j(n))$ such that $\alpha_{j(n+1)}>2 x_{n+1}, \omega\left(\alpha_{j(n+1)}\right) \geq 2^{n+2-i} \omega\left(x_{i}\right), 1 \leq i \leq n+1$, and $\omega(x) \leq$ $x /(n+1)^{2}$ if $x \geq \alpha_{j(n+1)}$. Now define $x_{n+2}:=\alpha_{j(n+1)}$. 
As in Braun, Meise, and Taylor [7, Lemma 1.6], define

$$
\sigma:\left[0, \infty\left[\rightarrow \left[0, \infty\left[, \quad \sigma(x):=n \omega(x)-\sum_{i=1}^{n} \omega\left(x_{i}\right), \quad x \in\left[x_{n}, x_{n+1}[.\right.\right.\right.\right.\right.
$$

Then it follows as in the proof of [7, Lemma 1.6] that $\sigma$ is continuous, has properties $(\alpha)$ and $(\delta)$ of 2.1, and $\omega(t)=o(\sigma(t))$ as $t \rightarrow \infty$, which implies that $\sigma$ has property $(\gamma)$ as well. On the other hand, for each $t \in\left[x_{n}, x_{n+1}\right]$, we get $\sigma(t) \leq n \omega(t) \leq t / n$, and therefore $\sigma(t)=o(t)$ as $t \rightarrow \infty$. Moreover, since $\omega$ satisfies condition $\left(\alpha_{1}\right)$ it follows from [7, Lemma 1.7] that $\sigma$ also satisfies $\left(\alpha_{1}\right)$.

To show that $\sigma\left(a_{j(k)}\right)=O\left(\left|\operatorname{Im} a_{j(k)}\right|\right)$ as $k \rightarrow \infty$, we first fix $k(0) \in \mathbb{N}$ such that $\sigma\left(a_{j(k)}\right) \geq 1$ if $k \geq k(0)$. Then we apply property 2.1( $\left.\alpha\right)$ for the weight function $\sigma$ to find $L \geq 1$ such that for all $k \geq k(0)$,

$$
\begin{aligned}
\sigma\left(a_{j(k)}\right) & \leq \sigma\left(3\left|\alpha_{j(k)}\right| / 2\right) \leq L \sigma\left(\alpha_{j(k)}\right)=L \sigma\left(x_{k+1}\right) \leq L k \omega\left(x_{k+1}\right) \\
& \leq L j(k) \omega\left(a_{j(k)}\right) \leq L j(k) \frac{1}{j(k)^{2}}\left|\operatorname{Im} a_{j(k)}\right| \leq L\left|\operatorname{Im} a_{j(k)}\right| .
\end{aligned}
$$

CASE 2: $\liminf _{j \rightarrow \infty}\left|\operatorname{Im} a_{j}\right| /\left|a_{j}\right|>0$. Then there are $\delta>0$ and a subsequence $\left(a_{j(k)}\right)_{k \in \mathbb{N}}$ such that $\left|\operatorname{Im} a_{j(k)}\right| \geq \delta\left|a_{j(k)}\right|$ for each $k \in \mathbb{N}$. Next choose inductively a sequence $\left(x_{n}\right)_{n \in \mathbb{N}}$ in $[0, \infty[$ which satisfies

$$
x_{1}=0, \quad x_{n+1} \geq 2 x_{n}, \quad \omega\left(x_{2}\right)>0, \quad \omega(t) / t \leq 1 / n^{2} \quad \text { if } t \geq x_{n},
$$

and

$$
\omega\left(x_{n+1}\right) \geq 2^{n+1-i} \omega\left(x_{i}\right), \quad 1 \leq i \leq n,
$$

and define $\sigma$ as in (3.1). Then $\sigma$ is a weight function which satisfies $\omega(t)=$ $o(\sigma(t)), \sigma(t)=o(t)$ as $t \rightarrow \infty$, and condition $\left(\alpha_{1}\right)$. Next choose $k_{0} \in \mathbb{N}$ such that $\sigma\left(a_{j(k)}\right) \geq 1$ if $k \geq k_{0}$. By the property $2.1(\beta)$ of the weight $\sigma$, we find $C \geq 1$ such that, for $k \geq k_{0}$,

$$
\sigma\left(a_{j(k)}\right) \leq C\left|a_{j(k)}\right| \leq \frac{C}{\delta}\left|\operatorname{Im} a_{j(k)}\right| .
$$

3.2. Proposition. Let $\omega$ be a weight function which satisfies $\left(\alpha_{1}\right)$ and let $\mu \in \mathcal{E}_{(\omega)}^{\prime}(\mathbb{R})$. If the convolution operator

$$
T_{\mu}: \mathcal{E}_{(\omega)}(\mathbb{R}) \rightarrow \mathcal{E}_{(\omega)}(\mathbb{R})
$$

admits a continuous linear right inverse, then the following two conditions are satisfied:

(a) $\widehat{\mu}$ is ( $\omega)$-slowly decreasing.

(b) There exists $C>0$ such that

$$
|\operatorname{Im} a| \leq C(1+\omega(a)), \quad a \in \mathbb{C}, \widehat{\mu}(a)=0 .
$$


Proof. If $T_{\mu}: \mathcal{E}_{(\omega)}(\mathbb{R}) \rightarrow \mathcal{E}_{(\omega)}(\mathbb{R})$ admits a continuous linear right inverse, then $T_{\mu}$ is surjective. Hence Momm's Theorem 2.8 implies that $\widehat{\mu}$ is $(\omega)$ slowly decreasing, which proves (a).

To prove that condition (b) holds we argue by contradiction and assume that it is not satisfied. Then there exists a sequence $\left(a_{j}\right)_{j \in \mathbb{N}}$ of complex numbers such that $\widehat{\mu}\left(a_{j}\right)=0$ and $\omega\left(a_{j}\right) \leq j^{-2}\left|\operatorname{Im} a_{j}\right|$ for each $j \in \mathbb{N}$. By Lemma 3.1 we can choose a weight function $\sigma$ with $\omega=o(\sigma)$, which also satisfies condition $\left(\alpha_{1}\right)$, such that by passing to a subsequence, we have $\sigma\left(a_{j}\right)=O\left(\left|\operatorname{Im} a_{j}\right|\right)$ as $j \rightarrow \infty$. Proceeding by recurrence, we extract a subsequence of $\left(a_{j}\right)_{j \in \mathbb{N}}$, which we denote in the same way, such that, for $n(t):=$ $\operatorname{card}\left\{j \in \mathbb{N}:\left|a_{j}\right| \leq t\right\}$,

(i) $\left|a_{j+1}\right| \geq 4\left|a_{j}\right|$,

(ii) $n(t) \log t=o(\omega(t))$ as $t \rightarrow \infty$.

As in Braun, Meise, and Vogt [8, 3.11], define

$$
F(z):=\prod_{j=1}^{\infty}\left(1-\frac{z}{a_{j}}\right), \quad z \in \mathbb{C} .
$$

By Rudin [28, Theorem 15.6], $F$ is an entire function such that its set of zeros consists of the sequence $\left(a_{j}\right)_{j \in \mathbb{N}}$, and satisfies the following conditions:

(1) There exists $C>0$ such that $|F(z)| \leq C \exp (\omega(z)), z \in \mathbb{C}$.

(2) There exists $\varepsilon_{0}>0$ such that $|F(\zeta)| \geq \varepsilon_{0} \exp (-\omega(\zeta))$ for all $\zeta \in$ $\mathbb{C} \backslash \bigcup_{j=1}^{\infty} B\left(a_{j}, 1\right)$.

(3) There exist $\varepsilon_{0}, K_{0}>0$ such that, if $\zeta \in \mathbb{C}$ satisfies $1 \leq\left|\zeta-a_{j}\right| \leq 2$ for some $j$, then

$$
|F(\zeta)| \geq \varepsilon_{0} \exp \left(-K_{0} \omega\left(a_{j}\right)\right)
$$

This can be achieved by the arguments given in [6, proof of Lemma 3.5], based on $[8,3.11]$. In particular, $F$ is $(\omega)$-slowly decreasing.

Since each $a_{j}$ is a zero of $\widehat{\mu}$, it follows that $g:=\widehat{\mu} / F$ is an entire function. Since $F$ is $(\omega)$-slowly decreasing, we conclude that $g \in A_{(\omega)}(\mathbb{C}, \mathbb{R})$ (notation as in 2.4). This implies, in particular, that $M_{g}: A_{(\omega)}(\mathbb{C}, \mathbb{R}) \rightarrow A_{(\omega)}(\mathbb{C}, \mathbb{R})$ is continuous.

By hypothesis $T_{\mu}: \mathcal{E}_{(\omega)}(\mathbb{R}) \rightarrow \mathcal{E}_{(\omega)}(\mathbb{R})$ admits a continuous linear right inverse. Therefore, $M_{\widehat{\mu}}: A_{(\omega)}(\mathbb{C}, \mathbb{R}) \rightarrow A_{(\omega)}(\mathbb{C}, \mathbb{R})$ admits a continuous linear left inverse $L_{\widehat{\mu}}$. The operator $L_{F}:=L_{\widehat{\mu}} \circ M_{g}: A_{(\omega)}(\mathbb{C}, \mathbb{R}) \rightarrow A_{(\omega)}(\mathbb{C}, \mathbb{R})$ is continuous, and it is a left inverse of $M_{F}$ since

$$
L_{F} M_{F}(h)=L_{\widehat{\mu}} M_{g} M_{F}(h)=L_{\widehat{\mu}}(g F h)=L_{\widehat{\mu}}(\widehat{\mu} h)=h, \quad h \in A_{(\omega)}(\mathbb{C}, \mathbb{R}) .
$$

Thus $M_{F}: A_{(\omega)}(\mathbb{C}, \mathbb{R}) \rightarrow A_{(\omega)}(\mathbb{C}, \mathbb{R})$ admits a continuous linear left inverse. 
Since the weight $\sigma$ constructed at the beginning of the proof by the use of Lemma 3.1 satisfies $\omega(t)=o(\sigma(t))$ as $t \rightarrow \infty$, we conclude that $A_{(\omega)}(\mathbb{C}, \mathbb{R}) \subset A_{\{\sigma\}}(\mathbb{C}, \mathbb{R})$ and that the inclusion map is continuous. Moreover, the growth estimate for $F$ in (1) implies that $M_{F}$ also defines a continuous linear operator from $A_{\{\sigma\}}(\mathbb{C}, \mathbb{R})$ into itself. Next define

$$
\varrho: H(\mathbb{C}) \rightarrow \mathbb{C}^{\mathbb{N}}, \quad \varrho(f):=\left(f\left(a_{j}\right)\right)_{j \in \mathbb{N}} .
$$

Proceeding as in the proof of [6, Lemma 3.8] (based on the method of Meise [15, Theorem 3.7]), we can conclude from the properties (1)-(3) of $F$ that

$$
\begin{aligned}
M_{F} A_{(\omega)}(\mathbb{C}, \mathbb{R}) & =\left\{f \in A_{(\omega)}(\mathbb{C}, \mathbb{R}): f\left(a_{j}\right)=0 \forall j \in \mathbb{N}\right\} \\
& =\operatorname{ker} \varrho \cap A_{(\omega)}(\mathbb{C}, \mathbb{R}), \\
M_{F} A_{\{\sigma\}}(\mathbb{C}, \mathbb{R}) & =\left\{f \in A_{\{\sigma\}}(\mathbb{C}, \mathbb{R}): f\left(a_{j}\right)=0 \forall j \in \mathbb{N}\right\} \\
& =\operatorname{ker} \varrho \cap A_{\{\sigma\}}(\mathbb{C}, \mathbb{R}) .
\end{aligned}
$$

Moreover, the map $\varrho$ defined above induces an isomorphism between the quotient $A_{(\omega)}(\mathbb{C}, \mathbb{R}) / M_{F} A_{(\omega)}(\mathbb{C}, \mathbb{R})$ and the sequence space

$$
E:=\left\{\left(x_{j}\right)_{j \in \mathbb{N}} \in \mathbb{C}^{\mathbb{N}}: \exists n:\|x\|_{n}:=\sup _{j \in \mathbb{N}}\left|x_{j}\right| \exp \left(-n\left|\operatorname{Im} a_{j}\right|-n \omega\left(a_{j}\right)\right)<\infty\right\},
$$

as well as an isomorphism between $A_{\{\sigma\}}(\mathbb{C}, \mathbb{R}) / M_{F} A_{\{\sigma\}}(\mathbb{C}, \mathbb{R})$ and the $(\mathrm{LF})$ sequence space

$$
G:=\operatorname{ind}_{\rightarrow n} \operatorname{proj}_{\leftarrow k} K(n, k),
$$

where

$$
K(n, k):=\left\{x \in \mathbb{C}^{\mathbb{N}}:\|x\|_{n, k}:=\sup _{j \in \mathbb{N}}\left|x_{j}\right| \exp \left(-n\left|\operatorname{Im} a_{j}\right|-\frac{1}{k} \sigma\left(a_{j}\right)\right)<\infty\right\} .
$$

We then obtain the diagram

$$
\begin{array}{r}
0 \rightarrow A_{(\omega)}(\mathbb{C}, \mathbb{R}) \stackrel{M_{F}}{\longrightarrow} A_{(\omega)}(\mathbb{C}, \mathbb{R}) \stackrel{\varrho_{1}}{\longrightarrow} E \rightarrow 0 \\
\cap \\
0 \rightarrow A_{\{\sigma\}}(\mathbb{C}, \mathbb{R}) \stackrel{M_{F}}{\longrightarrow} A_{\{\sigma\}}(\mathbb{C}, \mathbb{R}) \stackrel{\varrho_{2}}{\longrightarrow} G \rightarrow 0
\end{array}
$$

where $\varrho_{1}$ and $\varrho_{2}$ are the restrictions of $\varrho$. Since $M_{F}$ has a continuous linear left inverse, $\varrho_{1}$ has a continuous linear right inverse $R_{1}: E \rightarrow A_{(\omega)}(\mathbb{C}, \mathbb{R})$.

Next note that $\omega\left(a_{j}\right) \leq j^{-2}\left|\operatorname{Im} a_{j}\right|, j \in \mathbb{N}$, implies, for each $n \in \mathbb{N}$,

$$
0<n\left|\operatorname{Im} a_{j}\right| \leq n\left|\operatorname{Im} a_{j}\right|+n \omega\left(a_{j}\right) \leq\left(n+\frac{n}{j^{2}}\right)\left|\operatorname{Im} a_{j}\right| \leq 2 n\left|\operatorname{Im} a_{j}\right|,
$$

while the choice of $\sigma$ implies the existence of $C \in \mathbb{N}$ such that $\sigma\left(a_{j}\right) \leq$ 
$C\left|\operatorname{Im} a_{j}\right|+C, j \in \mathbb{N}$. Hence for each $n \in \mathbb{N}$ we have

$$
\begin{aligned}
0<n\left|\operatorname{Im} a_{j}\right| & \leq n\left|\operatorname{Im} a_{j}\right|+\frac{1}{k} \sigma\left(a_{j}\right) \leq n\left|\operatorname{Im} a_{j}\right|+\frac{C}{k}\left|\operatorname{Im} a_{j}\right|+\frac{C}{k} \\
& \leq(n+C)\left|\operatorname{Im} a_{j}\right|+C .
\end{aligned}
$$

From (3.2) and (3.3) it follows easily that the sequence spaces $E$ and $G$ coincide algebraically and topologically with the dual of $P:=\Lambda_{\infty}\left(\left(\left|\operatorname{Im} a_{j}\right|\right)_{j \in \mathbb{N}}\right)$. If we identify $E$ and $G$ and use the fact that $A_{(\omega)}(\mathbb{C}, \mathbb{R}) \subset A_{\{\sigma\}}(\mathbb{C}, \mathbb{R})$, then the map $R_{1}: E=G \rightarrow A_{\{\sigma\}}(\mathbb{C}, \mathbb{R})$ is a continuous linear right inverse for $\varrho_{2}$. Hence $G=P^{\prime}$ is isomorphic to a complemented subspace of $A_{\{\sigma\}}(\mathbb{C}, \mathbb{R})=\mathcal{E}_{\{\sigma\}}^{\prime}(\mathbb{R})$. Since $\sigma$ satisfies condition $\left(\alpha_{1}\right)$, a result of Vogt [30, Theorem 1.8], and Bonet and Domański [4, Theorem 6.2 in connection with Propositions 5.3(a) and 5.4(b)] implies that each Fréchet quotient of $\mathcal{E}_{\{\omega\}}(\mathbb{R})$ and hence $P$ has the topological invariant $(\overline{\bar{\Omega}})$. However, this is a contradiction since no power series space satisfies $(\overline{\bar{\Omega}})$. Hence condition (b) is satisfied.

Remark. For $\omega(t)=t$ we get $\mathcal{E}_{(\omega)}(\mathbb{R})=H(\mathbb{C})$. By Taylor $[29$, Theorem 5.1] or Meise [16, Theorem 3.5], each convolution operator on $H(\mathbb{C})$ admits a continuous linear right inverse. This is the reason why we require in $2.1(\beta)$ that $\omega(t)=o(t)$ as $t$ tends to infinity.

Next we show that the necessary conditions in Proposition 3.2 are sufficient if $\omega$ is a (DN)-weight function.

3.3. Proposition. Let $\omega$ be a (DN)-weight function. Then for $\mu \in$ $\mathcal{E}_{(\omega)}^{\prime}(\mathbb{R})$ the convolution operator

$$
T_{\mu}: \mathcal{E}_{(\omega)}(\mathbb{R}) \rightarrow \mathcal{E}_{(\omega)}(\mathbb{R})
$$

admits a continuous linear right inverse if conditions (a) and (b) in Proposition 3.2 hold.

Proof. Obviously, it is no restriction to assume that $V(\widehat{\mu}):=\{a \in \mathbb{C}:$ $\widehat{\mu}(a)=0\}$ is infinite. To simplify the notation we let

$$
F:=\widehat{\mu} \in A_{(\omega)}(\mathbb{C}, \mathbb{R}), \quad p(z):=|\operatorname{Im} z|+\omega(z) .
$$

Note that then $A_{(\omega)}(\mathbb{C}, \mathbb{R})$ coincides with the algebra $A_{p}(\mathbb{C})$ of Berenstein and Taylor [2] and Meise [15]. Since $F=\widehat{\mu}$ is $(\omega)$-slowly decreasing, it follows from Theorem 2.8 that there are $k, m \in \mathbb{N}$ and $R>0$ such that for each $a \in V(F),|a| \geq R$, there is a circle $T_{a}$ surrounding $a$ with diameter $d\left(T_{a}\right) \leq k \omega(a)+|\operatorname{Im} a|$ and $|F(w)| \geq \exp (-m p(w))$ for each $w$ in $T_{a}$. By condition (b), $d\left(T_{a}\right) \leq(k+C) \omega(a)+C$.

We apply again the fact that $F$ is $(\omega)$-slowly decreasing to find $0<$ $\varepsilon_{0}<1, C_{0}>0, D_{0}>0$ such that every component of $S\left(F, \varepsilon_{0}, C_{0}\right):=$ $\left\{z \in \mathbb{C}:|F(z)|<\varepsilon_{0} \exp \left(-C_{0} p(z)\right)\right\}$ (is bounded and) atisfies $\sup _{z \in S} p(z) \leq$ $D_{0}\left(1+\inf _{z \in S} p(z)\right)$. Without restriction, we may assume that $C_{0}>m$. 
Let $S$ be a connected component of $S\left(F, \varepsilon_{0}, C_{0}\right)$ which has a non-empty intersection with $V(F)$ and let $a \in S \cap V(F)$. If $|a| \geq R$, then $S$ is contained in the disc $D_{a}$ limited by the circle $T_{a}$. In particular, if $z \in S$, then

$$
|\operatorname{Im} z| \leq|\operatorname{Im} a|+d\left(T_{a}\right) \leq 2|\operatorname{Im} a|+k \omega(a) \leq(k+2 C) \omega(a)+2 C .
$$

On the other hand, if $z \in D_{a},|z-a| \leq d\left(T_{a}\right)$, and if $K_{0}$ is chosen according to condition 2.1 $(\alpha)$ such that $\omega(2 t) \leq K_{0}(\omega(t)+1)$ for $t \geq 0$, then

$$
\begin{aligned}
\omega(a) & \leq K_{0} \omega(z)+K_{0} \omega\left(d\left(T_{a}\right)\right)+K_{0}^{2} \\
& \leq K_{0} \omega(z)+K_{0} \omega(C+(k+C) \omega(a))+K_{0}^{2} \\
& \leq K_{0} \omega(z)+K_{0}^{2} \omega(C)+K_{0}^{2} \omega((k+C) \omega(a))+2 K_{0}^{2} .
\end{aligned}
$$

Thus

$$
\omega(a)\left(1-(k+C) K_{0}^{2} \frac{\omega((k+C) \omega(a))}{(k+C) \omega(a)}\right) \leq K_{0} \omega(z)+K_{0}^{2} \omega(C)+2 K_{0}^{2} .
$$

Since $\omega(t)=o(t)$, if $|a| \geq R_{1}>R$, we get $\omega(a) \leq 2 K_{0} \omega(z)+2 K_{0}^{2} \omega(C)+4 K_{0}^{2}$, and the existence of $A_{1}>0$ such that $|\operatorname{Im} z| \leq 2(k+2 C) K_{0} \omega(z)+A_{1}$ for each $z \in S$.

Since there are only finitely many components $S$ which meet $V(F)$ at a point $a$ with $|a| \leq R_{1}$, and they are bounded, we conclude that there is $A_{2} \geq 1$ such that for each component $S$ of $S\left(F, \varepsilon_{0}, C_{0}\right)$ which satisfies $S \cap V(F) \neq \emptyset$ we have

$$
|\operatorname{Im} z| \leq A_{2} \omega(z)+A_{2}, \quad z \in S .
$$

Denote by $I_{\text {loc }}(F)$ the ideal of $A_{(\omega)}(\mathbb{C}, \mathbb{R})$ of all $g \in A_{(\omega)}(\mathbb{C}, \mathbb{R})$ such that $V(g) \supset V(F)$ and at each $a \in V(F)$ the order of vanishing of $g$ is at least as high as the one of $F$ at $a$, i.e., $\operatorname{ord}(g, a) \geq \operatorname{ord}(F, a)$.

Since $F$ is $(\omega)$-slowly decreasing, $I_{\text {loc }}(F)=F A_{(\omega)}(\mathbb{C}, \mathbb{R})$ and it is closed; see [2] and [15, 3.5]. We select the connected components of $S\left(F, \varepsilon_{0}, C_{0}\right)$ which intersect $V(F)$ and label them as $\left(S_{j}\right)_{j \in \mathbb{N}}$ so that $\gamma_{j}:=\sup _{z \in S_{j}} p(z)$, $j \in \mathbb{N}$, is increasing. Recall that there is $D_{1}>0$ such that

$$
\omega(z) \leq p(z) \leq D_{1}+D_{1} \omega(z), \quad z \in S_{j}, j \in \mathbb{N} .
$$

Put $\gamma=\left(\gamma_{j}\right)_{j \in \mathbb{N}}$. Following the proof of [15, Theorem 3.7, pp. 77-78] we let

$$
E_{j}:=\prod_{b \in S_{j} \cap V(F)} \mathbb{C}^{\operatorname{ord}(F, b)}, \quad j \in \mathbb{N},
$$

and we define $\varrho_{j}: H^{\infty}\left(S_{j}\right) \rightarrow E_{j}$ by

$$
\varrho_{j}(f):=\left(\left(\frac{1}{k !} f^{(k)}(b)\right)_{0 \leq k<\operatorname{ord}(F, b)}\right)_{b \in S_{j} \cap V(F)} .
$$


We endow $E_{j}$ with the quotient norm

$$
\left\|\varrho_{j}(g)\right\|:=\inf \left\{\|h\|_{H^{\infty}\left(S_{j}\right)}: \varrho_{j}(h)=\varrho_{j}(g)\right\}, \quad g \in H^{\infty}\left(S_{j}\right) .
$$

Then $\varrho_{j}$ is linear, continuous and surjective. Now, for each $j \in \mathbb{N}$ there is $R_{j}: E_{j} \rightarrow H^{\infty}\left(S_{j}\right)$ continuous and linear such that $\varrho_{j} R_{j}=\operatorname{id}_{E_{j}}$ and $\left\|R_{j}\right\| \leq$ $2 \operatorname{dim} E_{j}$. If $f \in A_{(\omega)}(\mathbb{C}, \mathbb{R})$, then $\|f\|_{n}:=\sup _{z \in \mathbb{C}}|f(z)| \exp (-n p(z))<\infty$ for some $n \in \mathbb{N}$. This implies

$$
\left\|\left.f\right|_{S_{j}}\right\|_{H^{\infty}\left(S_{j}\right)} \leq e^{n \gamma_{j}}\|f\|_{n}
$$

Therefore, $\left\|\varrho_{j}\left(\left.f\right|_{S_{j}}\right)\right\|_{j} \leq e^{n \gamma_{j}}\|f\|_{n}$ and consequently $\left(\varrho_{j}\left(\left.f\right|_{S_{j}}\right)\right)_{j \in \mathbb{N}}$ is in $K_{\infty}\left(\gamma,\left(E_{j}\right)_{j}\right)$, where

$$
K_{\infty}\left(\gamma,\left(E_{j}\right)_{j}\right):=\left\{\left(x_{j}\right)_{j} \in \prod_{j \in \mathbb{N}} E_{j}: \exists n:\|\| x\left\|_{n}:=\sup _{j \in \mathbb{N}}\right\| x_{j} \|_{j} e^{-n \gamma_{j}}<\infty\right\} .
$$

By the above, the map

$$
\varrho: A_{(\omega)}(\mathbb{C}, \mathbb{R}) \rightarrow K_{\infty}\left(\gamma,\left(E_{j}\right)_{j \in \mathbb{N}}\right), \quad \varrho(g):=\left(\varrho_{j}\left(\left.g\right|_{S_{j}}\right)\right)_{j \in \mathbb{N}}
$$

is linear and continuous. The arguments of Meise [15, pp. 77-78], which involve the semilocal to global extension theorem of Berenstein and Taylor $[3,2.2]$ (see also $\left[2\right.$, p. 110]), show that $\varrho$ is surjective and $\operatorname{ker} \varrho=I_{\text {loc }}(F)$. In particular, $K_{\infty}\left(\gamma,\left(E_{j}\right)_{j \in \mathbb{N}}\right)$ is nuclear, so we can apply $[15,1.3]$ to conclude that for each $n \in \mathbb{N}$ there exist $k \in \mathbb{N}$ and $d>0$ such that

$$
2\left(\operatorname{dim} E_{j}\right) e^{n \gamma_{j}} \leq d e^{k \gamma_{j}}, \quad j \in \mathbb{N} .
$$

We want to show that $\varrho$ has a continuous linear right inverse. To do this, fix $y=\left(y_{j}\right)_{j \in \mathbb{N}} \in K_{\infty}\left(\gamma,\left(E_{j}\right)_{j}\right)$. Then there is $n \in \mathbb{N}$ with $\|y\|_{n}:=$ $\sup _{j \in \mathbb{N}}\left\|y_{j}\right\|_{j} e^{-n \gamma_{j}}<\infty$. Now $\lambda_{j}:=R_{j} y_{j}$ is in $H^{\infty}\left(S_{j}\right)$ and satisfies $\left\|\lambda_{j}\right\|_{H^{\infty}\left(S_{j}\right)}$ $\leq 2\left(\operatorname{dim} E_{j}\right)\left\|y_{j}\right\|_{j} \leq 2\left(\operatorname{dim} E_{j}\right)\left|\|y \mid\|_{n} e^{n \gamma_{j}}\right.$. By (3.7) this implies

$$
\left\|\lambda_{j}\right\|_{H^{\infty}\left(S_{j}\right)} \leq d\|\| y\|\|_{n} e^{k \gamma_{j}} \leq d\|\| y \mid \|_{n} \exp \left(k D_{0}\left(1+\inf _{z \in S_{j}} p(z)\right)\right) .
$$

Therefore, we deduce by (3.4) that for each $z \in S_{j}$ we have

$$
\left|\lambda_{j}(z)\right| \leq d e^{k D_{0}}\||y|\|_{n} \exp \left(k D_{0} p(z)\right) \leq d e^{k D_{0}+k D_{0} D_{1}} \mid\|y\|_{n_{n}} \exp \left(k D_{0} D_{1} \omega(z)\right) .
$$

We define $P(y) \in H\left(S\left(F, \varepsilon_{0}, C_{0}\right)\right)$ by $P(y)(z)=R_{j} y_{j}(z)$ if $z \in S_{j}$ and $P(y)(z)=0$ otherwise. Clearly, $P: K_{\infty}\left(\gamma,\left(E_{j}\right)_{j}\right) \rightarrow H\left(S\left(F, \varepsilon_{0}, C_{0}\right)\right)$ is well-defined and linear. Moreover,

$$
|P(y)(z)| \leq d e^{k\left(D_{0}+D_{0} D_{1}\right)}\|\| y \|_{n} \exp \left(k D_{0} D_{1} \omega(z)\right), \quad z \in S\left(F, \varepsilon_{0}, C_{0}\right) .
$$

As in the proof of Bernstein and Gay [1, Theorem 2.1.4], it follows that there exist $0<\varepsilon_{1}<\varepsilon_{0}, C_{1}>C, A_{0}>0, B_{0}>0$, and $\chi \in C^{\infty}(\mathbb{C})$ satisfying $0 \leq \chi \leq 1, \chi \equiv 1$ on $S\left(F, \varepsilon_{1}, C_{1}\right)$, supp $\chi \subset S\left(F, \varepsilon_{0}, C_{0}\right)$ and

$$
\left|\frac{\partial \chi}{\partial \bar{z}}(z)\right| \leq A_{0} \exp \left(B_{0} p(z)\right), \quad z \in \mathbb{C} .
$$


Then $\chi P(y)$ is in $C^{\infty}(\mathbb{C})$ and is holomorphic on $S\left(F, \varepsilon_{1}, C_{1}\right)$. This implies $\frac{\partial}{\partial \bar{z}}(\chi P(y))=\frac{\partial \chi}{\partial \bar{z}} P(y)$. We set

$$
v:=-\frac{1}{F} \frac{\partial}{\partial \bar{z}}(\chi P(y))=-\frac{1}{F} \frac{\partial \chi}{\partial \bar{z}} P(y)
$$

Clearly, $v$ is a $C^{\infty}$-function on $\mathbb{C}$ which vanishes on $S\left(F, \varepsilon_{1}, C_{1}\right)$. To derive an estimate for $v$, note that $|F(z)| \geq \varepsilon_{1} \exp \left(-C_{1} p(z)\right)$ whenever $z \notin$ $S\left(F, \varepsilon_{1}, C_{1}\right)$ for some $j \in \mathbb{N}$. From this estimate and (3.8) as well as (3.4) we get the existence of $A_{3}, A_{4}, B_{1}, B_{2}>0$ such that

$$
|v(z)| \leq A_{3} e^{B_{1} p(z)} \frac{1}{\varepsilon_{1}} e^{C_{1} p(z)} A_{0} e^{B_{0} p(z)} \leq A_{4} e^{B_{2} \omega(z)} .
$$

To use this estimate we let

$$
K(\omega):=\left\{f \in C^{\infty}(\mathbb{C}):\|f\|_{k}:=\sup _{|\alpha| \leq k} \sup _{z \in \mathbb{C}}\left|f^{(\alpha)}(z)\right| e^{k \omega(z)}<\infty \forall k \in \mathbb{N}\right\} .
$$

Then $K(\omega)$ is a nuclear Fréchet space. Since $\omega$ is a (DN)-weight function, it follows from Meise and Taylor [18, Proposition 1.9 and Theorem 2.17] that

$$
0 \rightarrow A_{(\omega)} \rightarrow K^{\prime}(\omega) \stackrel{\bar{\partial}}{\rightarrow} K^{\prime}(\omega) \rightarrow 0
$$

is an exact sequence which splits. Hence there exists a continuous linear operator $L: K^{\prime}(\omega) \rightarrow K^{\prime}(\omega)$ which satisfies $\bar{\partial} \circ L=\operatorname{id}_{K^{\prime}(\omega)}$. Now note that the estimate (3.9) and the fact that $\omega$ satisfies condition $2.1(\gamma)$ imply that $v$ defines an element of $K^{\prime}(\omega)$ by

$$
v(f):=\int_{\mathbb{C}} v(z) f(z) d z, \quad f \in K(\omega) .
$$

Next we define

$$
Q: K_{\infty}\left(\gamma,\left(E_{j}\right)_{j \in \mathbb{N}}\right) \rightarrow K^{\prime}(\omega), \quad Q(y):=\chi P(y)+F L(v) .
$$

Then it follows from (3.8) and an easy computation that $Q$ is well-defined, linear and continuous. Moreover,

$$
\frac{\partial}{\partial \bar{z}} Q(y)=\frac{\partial \chi}{\partial \bar{z}} P(y)+F \frac{\partial}{\partial \bar{z}} L(v)=\frac{\partial \chi}{\partial \bar{z}} P(y)+F v=0 .
$$

Hence $Q(y) \in \operatorname{ker} \bar{\partial}$ and by (3.10) this implies $Q(y) \in A_{(\omega)}$. Since the sequence (3.10) is exact and consists of (DFN)-spaces, it follows from Meise and Vogt [22, Propositions 26.4 and 26.24] that it is topologically exact. Hence $Q$ is in fact a continuous linear map from $K_{\infty}\left(\gamma,\left(E_{j}\right)_{j \in \mathbb{N}}\right)$ into $A_{(\omega)}$. Since $A_{(\omega)} \subset A_{(\omega)}(\mathbb{C}, \mathbb{R})$ with continuous inclusion,

$$
Q: K_{\infty}\left(\gamma,\left(E_{j}\right)_{j}\right) \rightarrow A_{(\omega)}(\mathbb{C}, \mathbb{R})
$$

is linear, continuous, and satisfies $\varrho(Q(y))=y$ for each $y$ in $K_{\infty}\left(\gamma,\left(E_{j}\right)_{j}\right)$. In particular, $\operatorname{ker} \varrho=F A_{(\omega)}(\mathbb{C}, \mathbb{R})$ is complemented in $A_{(\omega)}(\mathbb{C}, \mathbb{R})$. 
To complete the proof, we must show that $T_{\mu}$ has a continuous linear right inverse. It is enough to see that $T_{\mu}^{t}: \mathcal{E}_{(\omega)}^{\prime}(\mathbb{R}) \rightarrow \mathcal{E}_{(\omega)}^{\prime}(\mathbb{R})$ has a continuous linear left inverse. Since $\mathcal{F} \circ T_{\mu}^{t}=M_{\widehat{\mu}} \circ \mathcal{F}$ for $M_{\widehat{\mu}} g:=\widehat{\mu} g$, we have proved that the image of $M_{\widehat{\mu}}$ is $\widehat{\mu} A_{(\omega)}(\mathbb{C}, \mathbb{R})=F A_{(\omega)}(\mathbb{C}, \mathbb{R})$, and that it is complemented in $A_{(\omega)}(\mathbb{C}, \mathbb{R})$. This yields the conclusion.

3.4. TheOREM. Let $\omega$ be a quasianalytic (DN)-weight function which satisfies condition $\left(\alpha_{1}\right)$. Then for $\mu \in \mathcal{E}_{(\omega)}^{\prime}(\mathbb{R})$ the convolution operator

$$
T_{\mu}: \mathcal{E}_{(\omega)}(\mathbb{R}) \rightarrow \mathcal{E}_{(\omega)}(\mathbb{R})
$$

admits a continuous linear right inverse if and only if conditions (a) and (b) in Proposition 3.2 hold.

Proof. This follows immediately from Propositions 3.2 and 3.3 .

REMARK. Note that Theorem 3.4 extends the main result of Meise and Vogt [21] from the non-quasianalytic to the quasianalytic case. In the nonquasianalytic case, however, it was not required that $\omega$ is a $(\mathrm{DN})$-weight function, nor that $\omega$ satisfies $\left(\alpha_{1}\right)$. In fact, the theorem holds for $\omega(t)=$ $(\log (1+t))^{\alpha}, \alpha>1$, which is not a (DN)-weight function, however, it satisfies condition $\left(\alpha_{1}\right)$.

4. Ultradifferential operators on compact intervals. In this section we prove that $(\omega)$-ultradifferential operators can behave differently on the real line than on compact intervals. Also, we show that for them the characterization in Theorem 3.4 can be complemented by a condition which is similar to the one which was given by Domański and Vogt $[9$, Theorem 4.7]. To do so we need two lemmas and more notation.

4.1. Definition. Let $\omega$ be a weight function and assume that for $\mu \in$ $\mathcal{E}_{(\omega)}^{\prime}(\mathbb{R})$ its Fourier-Laplace transform $\widehat{\mu}$ is in $A_{(\omega)}$. Then the operator $T_{\mu}$ will be called an $(\omega)$-ultradifferential operator since for each $f \in \mathcal{E}_{(\omega)}(\mathbb{R})$ we have

$$
T_{\mu}(f)=\sum_{j=0}^{\infty} i^{j} \frac{\widehat{\mu}^{(j)}(0)}{j !} f^{(j)} .
$$

4.2. Lemma. Let $\omega$ be a weight function. Assume that $F \in A_{(\omega)}$ satisfies

$$
|F(z)| \leq B \exp (B \omega(z)), \quad z \in \mathbb{C},
$$

for some $B>0$ and that $F$ is $(\omega)$-slowly decreasing. Then there exist $m>0$ and $R_{0}>0$ such that for each $z \in \mathbb{C}$ with $|z| \geq R_{0}$ there exists $\zeta \in \mathbb{C}$ with $|z-\zeta| \leq m \omega(z)$ such that

$$
|F(\zeta)| \geq \exp (-m \omega(\zeta))
$$


Proof. Since $F$ is slowly decreasing, it follows from Theorem 2.8(3) that there exist $k, x_{0}>0$ such that for each $x \in \mathbb{R}$ with $|x| \geq x_{0}$ there exists $t \in \mathbb{R}$ with $|t-x| \leq k \omega(x)$ such that

$$
|F(t)| \geq \exp (-k \omega(t)) .
$$

Next we fix $z=x+i y$ in $\mathbb{C}$ with $|x| \geq x_{0}$ and consider two cases:

CASE 1: $|y| \leq k \omega(x)$. Since $|x| \geq x_{0}$ we can choose $t \in \mathbb{R}$ according to 2.8(3). Then $\zeta:=t$ satisfies

$$
|\zeta-z|=|t-x-i y| \leq|t-x|+|y| \leq 2 k \omega(x) \leq 2 k \omega(z) .
$$

Hence the assertion follows for $m=2 k$ in this case.

CASE 2: $|y|>k \omega(x)$. Select $n>2$ (depending on $z$ ) with $|y| / n<k \omega(x)$ and $t \in \mathbb{R}$ according to 2.8(3). We apply the minimum-modulus theorem [14, Chap. I, Theorem 11] with

$$
\eta:=\frac{1-\left(1+\frac{1}{\sqrt{2} n}\right)^{-1}}{16}
$$

to find $\varrho>0$ with $|t-z|<\varrho<\left(1+\frac{1}{\sqrt{2} n}\right)|t-z|=: R$ such that for $H=2+\log (3 e / 2 \eta)$ we have

$$
|F(\xi)| \geq|F(t)|^{H+1}\left(\sup _{|w-t|=2 e R}|F(w)|\right)^{-H}
$$

for each $\xi \in \mathbb{C}$ with $|\xi-t|=\varrho$. We now choose $\zeta \in \mathbb{C}$ with $|\zeta-t|=\varrho$ and $|\zeta-z|<|t-z| /(\sqrt{2} n)$. Then

$$
\begin{aligned}
|\zeta-z| & <\frac{|t-z|}{\sqrt{2} n}=\frac{\left(|t-x|^{2}+|y|^{2}\right)^{1 / 2}}{\sqrt{2} n} \leq \frac{\left((k \omega(x))^{2}+|y|^{2}\right)^{1 / 2}}{\sqrt{2} n}<\frac{|y|}{n} \\
& \leq k \omega(x) \leq k \omega(z) .
\end{aligned}
$$

To derive the desired lower bound for $F(\zeta)$ we estimate $\omega(\xi)$ for $|\xi-t| \leq 2 e R$ (in particular for $\xi=t$ ). To do this, we note first that by $2.1(\alpha)$ there exists $K \geq 1$ such that for each $a, b \in \mathbb{R}$ we have

$$
\omega(a+b) \leq K(\omega(a)+\omega(b)+1), \quad \omega(2 a) \leq K \omega(a)+K .
$$

This implies

$\omega(\xi) \leq \omega(|\xi-t|+|t|) \leq K \omega(t)+K \omega(|\xi-t|)+K \leq K \omega(t)+K \omega(2 e R)+K$.

On the other hand, it follows from (4.3) that $\omega(t) \leq K \omega(|t-z|)+$ $K \omega(z)+K$. From the hypotheses in the present case we get

$$
|t-z| \leq|t-x|+|y| \leq k \omega(x)+|y| \leq 2|y|
$$

and hence

$$
\omega(|t-z|) \leq \omega(2|y|) \leq K \omega(y)+K .
$$


This implies

$$
\omega(t) \leq K^{2} \omega(y)+K \omega(z)+K^{2}+K \leq 2 K^{2} \omega(z)+2 K^{2}
$$

and consequently

$$
\omega(\xi) \leq 2 K^{3} \omega(z)+2 K^{3}+K \omega(2 e R)+K .
$$

From $2 e R \leq 12|t-z|$ and (4.4) we get

$\omega(2 e R) \leq \omega(12|t-z|) \leq \omega\left(2^{4}|t-z|\right) \leq K^{4} \omega(|t-z|)+4 K^{4} \leq K^{5} \omega(z)+5 K^{5}$.

Therefore,

$$
\omega(\xi) \leq 2 K^{3} \omega(z)+K^{6} \omega(z)+2 K^{3}+5 K^{6}+K \leq 3 K^{6} \omega(z)+8 K^{6} .
$$

On the other hand, $|z-\zeta| \leq|y| / n$ and

$$
|y|=|\operatorname{Im} z| \leq|\operatorname{Im} \zeta|+|\operatorname{Im}(z-\zeta)| \leq|\operatorname{Im} \zeta|+|z-\zeta| \leq|\operatorname{Im} \zeta|+|y| / n
$$

imply

$$
|y| \leq \frac{n}{n-1}|\operatorname{Im} \zeta| \leq 2|\operatorname{Im} \zeta|,
$$

and consequently $|y| / n \leq 2|\operatorname{Im} \zeta| / n \leq|\operatorname{Im} \zeta|$. Therefore, we have

$$
\begin{aligned}
\omega(z) & \leq K \omega(\zeta)+K \omega(|\zeta-z|)+K \leq K \omega(\zeta)+K \omega(|y| / n)+K \\
& \leq K \omega(\zeta)+K \omega(|\operatorname{Im} \zeta|)+K \leq 2 K \omega(\zeta)+K
\end{aligned}
$$

Since $|\zeta-t|=\varrho$, it follows from (4.2) together with (4.5)-(4.7) that

$$
\begin{aligned}
|F(\zeta)| \geq & |F(t)|^{H+1}\left(\max _{|\xi-t|=2 e R}|F(\xi)|\right)^{-H} \\
\geq & \exp (-(H+1) k \omega(t))\left[\max _{|\xi-t|=2 e R} B \exp (B \omega(\xi))\right]^{-H} \\
\geq & B^{-H} \exp (-(H+1) k \omega(t))\left[\exp \left(3 B K^{6} \omega(z)+8 B K^{6}\right)\right]^{-H} \\
\geq & B^{-H} \exp \left(-H 8 B K^{6}\right) \exp \left(-(H+1) k \omega(t)-3 B K^{6} H \omega(z)\right) \\
\geq & B^{-H} \exp \left(-8 H B K^{6}\right) \\
& \times \exp \left(-2(H+1) k K^{2} \omega(z)-3 B K^{6} H \omega(z)-2(H+1) k K^{2}\right) \\
\geq & B^{-H} \exp \left(-8 H B K^{6}-2(H+1) k K^{2}\right) \\
& \times \exp \left(-\left(2(H+1) k K^{2}+3 B K^{6} H\right) \omega(z)\right) \\
\geq & B^{-H} \exp \left(-8 H B K^{6}-2(H+1) k K^{2}\right. \\
& \left.-\left[2(H+1) k K+3 B K^{6} H\right](2 k \omega(\zeta)+K)\right) .
\end{aligned}
$$

Hence there exists $d_{1}$ independent of $z$ (very small) and $l$ large, depending on $B, k$, and $K$, but not on $z$, such that $|F(\zeta)| \geq d_{1} \exp (-l \omega(\zeta))$. Selecting $R_{1}>0$ such that $\exp (-\omega(\zeta))<d_{1}$ if $|\zeta| \geq R_{1}$ we conclude that

$$
|F(\zeta)| \geq \exp (-(l+1) \omega(\zeta)) \text {. }
$$

Hence the assertion follows for $m=\max (k, l+1)$ in this case. 
For $z=x+i y \in \mathbb{C}$ satisfying $|x| \leq x_{0}$ let $\widetilde{z}:=x_{0}+i y$ and choose $\zeta \in \mathbb{C}$ for $\widetilde{z}$ according to what we proved so far. Then

$$
|\zeta-z| \leq|\zeta-\widetilde{z}|+|\widetilde{z}-z| \leq m \omega(\widetilde{z})+\left|x_{0}-x\right| \leq m \omega(\widetilde{z})+2\left|x_{0}\right| .
$$

Note that by (4.3) we also have

$$
\omega(\widetilde{z}) \leq K \omega(z)+K \omega\left(2 x_{0}\right)+K .
$$

Since $\lim _{t \rightarrow \infty} \omega(t)=\infty$ this shows that there is $m^{\prime}>m$ such that the statement of the lemma holds with $m$ replaced by $m^{\prime}$.

4.3. Lemma. Let $\omega$ be a weight function and assume that $F \in A_{(\omega)}$ satisfies the hypotheses of Lemma 4.2. Then there exist positive numbers $\varepsilon_{0}$, $C_{0}$, and $D$ such that each component $S$ of

$$
S_{\omega}\left(F, \varepsilon_{0}, C_{0}\right):=\left\{z \in \mathbb{C}:|F(z)|<\varepsilon_{0} \exp \left(-C_{0} \omega(z)\right)\right\}
$$

satisfies

$$
\operatorname{diam} S \leq D \inf _{z \in S} \omega(z)+D .
$$

Proof. By Lemma 4.2 there exist $m>0$ and $R_{0}>0$ such that the conclusion of that lemma holds. We fix $z \in \mathbb{C}$ with $|z| \geq R_{0}$ and choose $\zeta=\zeta(z) \in \mathbb{C}$ with $|\zeta-z| \leq m \omega(z)$ such that

$$
|F(\zeta)| \geq \exp (-m \omega(\zeta)) \text {. }
$$

In order to apply the minimum-modulus theorem we let

$$
\eta:=\frac{1}{32}, \quad H:=2+\log \left(\frac{3 e}{2 \eta}\right), \quad R:=2|\zeta-z| .
$$

Then there exists $\varrho$ with $|\zeta-z|<\varrho<2|\zeta-z|$ such that

$$
|F(\xi)| \geq|F(\zeta)|^{H+1}\left(\sup _{|w-\zeta|=2 e R}|F(w)|\right)^{-H}
$$

for each $\xi \in \mathbb{C}$ with $|\xi-\zeta|=\varrho$. Next choose $\delta>0$ so small that $\delta \leq 1 / 28 m$. Since $\omega(t)=o(t)$ by $2.1(\beta)$, we may assume that $R_{0}$ is so large that

$$
\omega(t) \leq \delta t \quad \text { for } t \geq R_{0} .
$$

Now fix any $\xi \in \mathbb{C}$ with $|\xi-\zeta|=\varrho$ and note that by our choices we have

$$
|z| \leq|z-\zeta|+|\zeta-\xi|+|\xi| \leq 3 m \omega(z)+|\xi| \leq \frac{1}{2}|z|+|\xi|
$$

and hence $|z| \leq 2|\xi|$. This implies

$$
|\zeta| \leq|\xi|+|\zeta-\xi| \leq|\xi|+2 m \omega(z) \leq|\xi|+\frac{1}{4}|z| \leq 2|\xi| .
$$

If we assume that $K \geq 1$ is a constant for which (4.3) holds, then it follows from this that

$$
\omega(\zeta) \leq K \omega(\xi)+K, \quad \xi \in \mathbb{C},|\zeta-\xi|=\varrho .
$$


Similarly we get for any $w \in \mathbb{C}$ with $|w-\zeta|=2 e R$ the estimate

$$
\begin{aligned}
|w| & \leq|\zeta|+2 e R \leq|\zeta|+12 m \omega(z) \leq|\xi|+|\zeta-\xi|+12 m \omega(z) \\
& \leq|\xi|+14 m \omega(z) \leq|\xi|+|z| / 2 \leq 2|\xi|
\end{aligned}
$$

and hence

$$
\omega(w) \leq K \omega(\xi)+K
$$

for each $w, \xi \in \mathbb{C}$ satisfying $|w-\zeta|=2 e R,|\xi-\zeta|=\varrho$. From this estimate together with (4.9) and (4.8) it follows that for each $\xi \in \mathbb{C}$ with $|\xi-\zeta|=\varrho$ we have the estimate

$$
\begin{aligned}
|F(\xi)| & \geq \exp (-m(H+1)(K \omega(\xi)+K)) \exp (-H(K \omega(\xi)+K)) \\
& =\exp (-[m(H+1)+H] K) \exp (-K(m(H+1)+1) \omega(\xi)) .
\end{aligned}
$$

If we let $\varepsilon_{0}:=\exp (-[m(H+1)+H] K)$ and $C_{0}:=K(m(H+1)+1)$ then it follows that for each $z_{0} \in S_{\omega}\left(F, \varepsilon_{0}, C_{0}\right)$ with $\left|z_{0}\right| \geq R_{0}$ the connected component $S$ of $S_{\omega}\left(F, \varepsilon_{0}, C_{0}\right)$ which contains $z_{0}$ is contained in the open disk $B\left(\zeta\left(z_{0}\right), 2 m \omega\left(z_{0}\right)\right)$. In particular, we have

$$
\operatorname{diam} S \leq 4 m \omega\left(z_{0}\right)
$$

By our choice of $\delta$, we have $4 m \omega\left(z_{0}\right) \leq\left|z_{0}\right| / 7$. Now we choose $z_{1} \in \bar{S}$ such that $\inf _{z \in S} \omega(z)=\omega\left(z_{1}\right)$. Then we get

$$
\left|z_{0}\right| \leq\left|z_{1}\right|+\left|z_{0}-z_{1}\right| \leq\left|z_{1}\right|+\operatorname{diam} S \leq\left|z_{1}\right|+\frac{1}{7}\left|z_{0}\right|
$$

and consequently $\left|z_{0}\right| \leq \frac{7}{6}\left|z_{1}\right| \leq 2\left|z_{1}\right|$. This implies

$$
\operatorname{diam} S \leq 4 m \omega\left(z_{0}\right) \leq 4 m \omega\left(2 z_{1}\right) \leq 4 m K \inf _{z \in S} \omega(z)+4 m K .
$$

If we choose $D=\max \left(r_{0}, 4 m K\right)$, then we deduce the estimate diam $S \leq$ $D \inf _{z \in S} \omega(z)+D$.

4.4. Definition. For a weight function $\omega$ and $R>1$ we define the space

$$
A_{(\omega, R)}:=\operatorname{ind}_{n \rightarrow} A([-R, R], n) .
$$

4.5. LEMmA. Let $\omega$ be a (DN)-weight function or a strong weight function. Then for each $R>0$ the following assertions hold:

(a) $\mathcal{F}: \mathcal{E}_{(\omega)}^{\prime}[-R, R] \rightarrow A_{(\omega, R)}$ is a linear topological isomorphism.

(b) $A_{(\omega, R)}$ is a (DFN)-space.

(c) $A_{(\omega, R)}^{\prime}$ has properties $(\mathrm{DN})$ and $(\Omega)$.

Proof. (a) This follows from the proof of Meise and Taylor [17, Proposition 3.6], since the proof of the surjectivity of the map $G$ defined in that proposition does not use the assumption that $\omega$ is a non-quasianalytic weight function.

(b) The properties of a weight function and well-known results imply that the (LB)-space $A_{(\omega, R)}$ is in fact a (DFN)-space. 
(c) $A_{(\omega, R)}^{\prime}$ has $(\Omega)$ by Meise and Taylor [19, Lemma 1.10(c)]. If $\omega$ is a (DN)-weight function, the proof of [19, Proposition 5.3] shows that $\mathcal{E}_{(\omega)}[-R, R]$ and hence $A_{(\omega, R)}^{\prime}$ has $(\mathrm{DN})$. If $\omega$ is a strong weight function, then $\mathcal{E}_{(\omega)}[-R, R]$ has (DN) by [19, Corollary 5.6].

4.6. Lemma. Let $\omega$ be a weight function. Then for $F \in A_{(\omega)}$ the following conditions are equivalent:

(1) $F$ is $(\omega)$-slowly decreasing.

(2) For each $R>0$ the multiplication operator

$$
M_{F}: A_{(\omega, R)} \rightarrow A_{(\omega, R)}, \quad M_{F}(g):=F g,
$$

is an injective topological homomorphism.

(3) There exist positive numbers $\varepsilon_{0}, C_{0}$, and $D$ such that for $S_{\omega}\left(F, \varepsilon_{0}, C_{0}\right)$ the conclusion of Lemma 4.3 holds.

Proof. $(1) \Rightarrow(2)$. Fix $R>0$ and note first that $A_{(\omega, R)}$ is a (DFN)-space by Lemma 4.5. Hence (2) follows from the Baernstein lemma (see Meise and Vogt [22, Proposition 26.26]) if we show that for each bounded set $B$ in $A_{(\omega, R)}$ the set $M_{F}^{-1}(B)$ is bounded. Since the sets

$$
B_{n}:=\left\{f \in A_{(\omega, R)}: \sup _{z \in \mathbb{C}}|f(z)| e^{-R|\operatorname{Im} z|-n \omega(z)} \leq 1\right\}, \quad n \in \mathbb{N},
$$

form a fundamental sequence of bounded sets in $A_{(\omega, R)}$, it suffices to show that $M_{F}^{-1}\left(B_{n}\right)$ is bounded for each $n \in \mathbb{N}$. To do so, fix $n \in \mathbb{N}$. Since $F$ is $(\omega)$-slowly decreasing by hypothesis, it follows from Lemma 4.2 that there exist $m, R_{0}>0$ such that for each $z \in \mathbb{C},|z| \geq R_{0}$, there exists $\zeta \in \mathbb{C}$ such that $|\zeta-z| \leq m \omega(z)$ and $|F(\zeta)| \geq \exp (-m \omega(\zeta))$. Since $F$ is in $A_{(\omega)}$, there exists $A>0$ such that

$$
|F(z)| \leq A \exp (A \omega(z)), \quad z \in \mathbb{C} .
$$

Next fix $h \in M_{F}^{-1}\left(B_{n}\right)$ and let $g:=F h \in B_{n}$. Then

$$
|g(z)| \leq \exp (R|\operatorname{Im} z|+n \omega(z)), \quad z \in \mathbb{C} .
$$

Now we apply Hörmander [11, Lemma 3.2] for $r=m \omega(z)>0$ to get, for $|z| \geq R_{0}$,

$$
|h(z)|=\left|\frac{g(z)}{F(z)}\right| \leq \frac{\sup _{|w-z| \leq 4 m \omega(z)}|g(w)| \sup _{|w-z| \leq 4 m \omega(z)}|F(w)|}{\left(\sup _{|w-z| \leq m \omega(z)}|F(w)|\right)^{2}}
$$

To derive further estimates from (4.12), note that $\lim _{t \rightarrow \infty} \omega(t) / t=0$ implies the existence of $t_{0}>0$ such that $\omega(t+4 m \omega(t)) \leq \omega(2 t)$ for $t \geq t_{0}$. Since $\omega$ satisfies $2.1(\alpha)$, this implies the existence of $K_{1} \geq K$ such that

$$
\omega(t+4 m \omega(t)) \leq K \omega(t)+K_{1}, \quad t>0 .
$$


Next note that for $\zeta=\zeta(z)$ we similarly get the existence of $C_{1} \geq 1$ such that

$$
|\zeta| \leq|z|+|\zeta-z| \leq|z|+m \omega(z) \leq 2|z|+C_{1} .
$$

By (4.3), this implies

$$
2 m \omega(\zeta) \leq 2 m \omega\left(2|z|+C_{1}\right) \leq 2 m\left(K^{2} \omega(z)+K\left(K+1+\omega\left(C_{1}\right)\right)\right) .
$$

Now from (4.12) by (4.10), (4.11), (4.13), and (4.14) we get

$$
\begin{aligned}
|h(z)| / A \leq & \exp \left(R|\operatorname{Im} z|+(4 R m+n K+A K) \omega(z)+K_{1}(n+A)\right) \\
& \times \exp (2 m \omega(\zeta)) \\
\leq & \exp \left(R|\operatorname{Im} z|+\left(4 R m+n K+A K+2 m K^{2}\right) \omega(z)\right) \\
& \times \exp \left(K_{1}(n+A)+2 m K\left(K+1+\omega\left(C_{1}\right)\right)\right) .
\end{aligned}
$$

Hence there exist $D_{1} \geq 1$ and $D_{2} \geq 1$, not depending on $h$, such that

$$
|h(z)| \leq D_{1} \exp \left(R|\operatorname{Im} z|+D_{2} \omega(z)\right), \quad z \in \mathbb{C},|z| \geq R_{0} .
$$

This estimate implies that we can enlarge the constant $D_{1}$ to have the inequality for all $z \in \mathbb{C}$. Since $h$ was an arbitrary element of $M_{F}^{-1}\left(B_{n}\right)$ we have proved that $M_{F}^{-1}\left(B_{n}\right)$ is bounded in $A_{(\omega, R)}$.

$(2) \Rightarrow(1)$. By Theorem 2.8, condition (1) is equivalent to

(4.15) For each bounded set $B$ in $A_{(\omega)}(\mathbb{C}, \mathbb{R})$ the set $M_{F}^{-1}(B)$ is bounded in $A_{(\omega)}(\mathbb{C}, \mathbb{R})$.

To show that (4.15) holds, let $B$ be any bounded set in $A_{(\omega)}(\mathbb{C}, \mathbb{R})$. Since $A_{(\omega)}(\mathbb{C}, \mathbb{R})$ is a $(\mathrm{DFN})$-space, it follows easily that there exists $R>0$ such that $B$ is contained in $A_{(\omega, R)}$ and bounded there. Now (2) implies that $M_{F}^{-1}(B)$ is bounded in $A_{(\omega, R)}$ and hence bounded in $A_{(\omega)}(\mathbb{C}, \mathbb{R})$.

$(1) \Rightarrow(3)$. This holds by Lemma 4.3 .

$(3) \Rightarrow(1)$. Obviously, (3) implies condition (3) in Theorem 2.8. Hence (1) holds.

4.7. Lemma. Let $\omega$ be a weight function and assume that $F \in A_{(\omega)}$ is ( $\omega)$-slowly decreasing. Then for each $R>0$ the space $A_{(\omega, R)} / M_{F} A_{(\omega, R)}$ is either finite-dimensional or isomorphic to the strong dual of a nuclear power series space of infinite type.

Proof. Fix $R>0$ and note that $M_{F}: A_{(\omega, R)} \rightarrow A_{(\omega, R)}$ has closed range by Lemma 4.6. Note also that $A_{(\omega, R)} / M_{F} A_{(\omega, R)}$ is finite-dimensional if and only if $F$ has only finitely many zeros. Therefore, we assume from now on that $V(F):=\{a \in \mathbb{C}: F(a)=0\}$ is an infinite set. Next we choose $\varepsilon_{0}, C_{0}$, and $D$ according to Lemma 4.3 and we label the components $S$ of $S_{\omega}\left(F, \varepsilon_{0}, C_{0}\right)$ which satisfy $S \cap V(F) \neq \emptyset$ in such a way that the sequence $\alpha$ defined by

$$
\alpha_{j}:=\sup _{z \in S_{j}} \omega(z), \quad j \in \mathbb{N},
$$


is increasing. Also, we define the sequence $\beta$ by

$$
\beta_{j}:=\sup _{z \in S_{j}}|\operatorname{Im} z|, \quad j \in \mathbb{N} .
$$

Then we define the spaces $E_{j}$ and the maps $\varrho_{j}: H^{\infty}\left(S_{j}\right) \rightarrow E_{j}$ as in (3.5) and (3.6). Moreover, we let

$$
\begin{aligned}
K_{\infty}\left(\alpha, \beta,\left(E_{j}\right)_{j \in \mathbb{N}}\right):= & \left\{\left(x_{j}\right)_{j \in \mathbb{N}} \in \prod_{j \in \mathbb{N}} E_{j}:\right. \\
& \left.\exists n \in \mathbb{N}:\|x \mid\|_{n}:=\sup _{j \in \mathbb{N}}\left\|x_{j}\right\|_{j} e^{-n \alpha_{j}-R \beta_{j}}<\infty\right\} .
\end{aligned}
$$

If $f \in A_{(\omega, R)}$ satisfies

$$
\|f\|_{n}=\sup _{z \in \mathbb{C}}|f(z)| \exp (-R|\operatorname{Im} z|-n \omega(z))<\infty
$$

then for each $j \in \mathbb{N}$ we have

$$
\left\|\left.f\right|_{S_{j}}\right\|_{H^{\infty}\left(S_{j}\right)} \leq\|f\|_{n} \exp \left(n \alpha_{j}+R \beta_{j}\right) .
$$

This implies that

$$
\left\|\varrho_{j}\left(\left.f\right|_{S_{j}}\right)\right\|_{j} e^{-n \alpha_{j}-R \beta_{j}} \leq\|f\|_{n}, \quad j \in \mathbb{N} .
$$

Consequently,

$$
\varrho: A_{(\omega, R)} \rightarrow K_{\infty}\left(\alpha, \beta,\left(E_{j}\right)_{j \in \mathbb{N}}\right), \quad \varrho(f):=\left(\varrho_{j}\left(\left.f\right|_{S_{j}}\right)\right)_{j \in \mathbb{N}},
$$

is a linear and continuous map.

Next we claim that ker $\varrho=M_{F} A_{(\omega, R)}$. To show this, note first that by the definition of $\varrho$ we obviously have $M_{F} A_{(\omega, R)} \subset$ ker $\varrho$. To prove the reverse inclusion, let $g \in \operatorname{ker} \varrho$. Then $g / F$ is an entire function. Using Lemma 4.3 and standard arguments, it follows as in the proof of Berenstein and Taylor $\left[2\right.$, Proposition 3] that $g / F \in A_{(\omega, R)}$ and hence $g \in M_{F} A_{(\omega, R)}$.

To show that $\varrho$ is surjective, we argue similarly to the proof of Theorem 3.7 in Meise [15]. Let $y=\left(y_{j}\right)_{j \in \mathbb{N}} \in K_{\infty}\left(\alpha, \beta,\left(E_{j}\right)_{j \in \mathbb{N}}\right)$ and choose $n \in \mathbb{N}$ such that $\|y\|_{n}<\infty$. By the definition of the norm in $E_{j}$ we can choose $\lambda_{j} \in H^{\infty}\left(S_{j}\right)$ with $\varrho_{j}\left(\lambda_{j}\right)=y_{j}$ so that

$$
\left\|\lambda_{j}\right\|_{H^{\infty}\left(S_{j}\right)} \leq 2\|\| y \|_{n} e^{n \alpha_{j}+R \beta_{j}}, \quad j \in \mathbb{N} .
$$

Next define $\lambda: S_{\omega}\left(F, \varepsilon_{0}, C_{0}\right) \rightarrow \mathbb{C}$ by $\lambda(z):=\lambda_{j}(z)$ if $z \in S_{j}$ and $\lambda(z):=0$ if $z \in S_{\omega}\left(F, \varepsilon_{0}, C_{0}\right) \backslash \bigcup_{j \in \mathbb{N}} S_{j}$. Then the diameter estimate from Lemma 4.3 and the definition of $\alpha$ and $\beta$ imply the existence of $D_{1}>0$ such that

$$
|\lambda(z)| \leq 2|||y| \|_{n} \exp \left(R|\operatorname{Im} z|+D_{1} n \omega(z)+D_{1}\right) .
$$

If $K$ is a constant for which the estimate (4.3) holds then it follows that

$$
\left|\frac{\partial F}{\partial z}(\zeta)\right| \leq e^{K} B \exp (K B \omega(\zeta)), \quad \zeta \in \mathbb{C} .
$$


From this and Lemma 4.3 it follows that there exist $\varepsilon_{1}, C_{1}, L>0$ such that for each $z \in S_{\omega}\left(F, \varepsilon_{1}, C_{1}\right)$ the distance to $\mathbb{C} \backslash S_{\omega}\left(F, \varepsilon_{0}, C_{0}\right)$ is at least as large as $L^{-1} \exp (-L \omega(z))$. Therefore, there are $A_{0}, B_{0}>0$ and $\chi \in C^{\infty}(\mathbb{C})$ having the following properties:

$$
0 \leq \chi \leq 1, \quad \chi \equiv 1 \text { on } S_{\omega}\left(F, \varepsilon_{1}, C_{1}\right), \quad \operatorname{supp} \chi \subset S_{\omega}\left(F, \varepsilon_{0}, C_{0}\right),
$$

$$
\left|\frac{\partial \chi}{\partial \bar{z}}(z)\right| \leq A_{0} \exp \left(B_{0} \omega(z)\right), \quad z \in \mathbb{C} \text {. }
$$

Next let

$$
v:=-\frac{1}{F} \frac{\partial}{\partial \bar{z}}(\chi \lambda)=-\frac{1}{F} \frac{\partial \chi}{\partial \bar{z}} \lambda
$$

and note that $v$ is in $C^{\infty}(\mathbb{C})$ and vanishes on $S\left(F, \varepsilon_{1}, C_{1}\right)$. The estimates (4.16) and (4.17) imply that

$$
\begin{aligned}
|v(z)| & \leq \frac{1}{\varepsilon_{1}} A_{0} \cdot 2\left|\|y \mid\|_{n} e^{D_{1}} \exp \left(R|\operatorname{Im} z|+\left(C_{1}+B_{0}+D_{1} n\right) \omega(z)\right)\right. \\
& =A_{1} \exp \left(R|\operatorname{Im} z|+B_{1} \omega(z)\right), \quad z \in \mathbb{C},
\end{aligned}
$$

for suitable numbers $A_{1}, B_{1}>0$. Since $\omega$ satisfies condition $2.1(\gamma)$, it follows from this that

$$
\int_{\mathbb{C}}\left[|v(z)| \exp \left(-R|\operatorname{Im} z|-\left(B_{1}+1\right) \omega(z)\right)\right]^{2} d z<\infty .
$$

By Hörmander [12, Theorem 4.4.2], there exists $g \in L_{\text {loc }}^{2}(\mathbb{C})$ satisfying $\partial g / \partial \bar{z}=v$ and

$$
\int_{\mathbb{C}}\left[|g(z)| \exp \left(-R|\operatorname{Im} z|-\left(B_{1}+1\right) \omega(z)-\log \left(1+|z|^{2}\right)\right)\right]^{2} d z<\infty .
$$

Since $v$ is in $C^{\infty}(\mathbb{C})$ and since $\partial / \partial \bar{z}$ is elliptic, $g$ is in $C^{\infty}(\mathbb{C})$. Hence

$$
f:=\chi \cdot \lambda+g F
$$

is in $C^{\infty}(\mathbb{C})$ and satisfies

$$
\frac{\partial f}{\partial \bar{z}}=\frac{\partial \chi}{\partial \bar{z}} \lambda+\frac{\partial g}{\partial \bar{z}} F=0
$$

by the choice of $g$. Consequently, $f \in H(\mathbb{C})$ and the estimates $(4.16)$ and (4.18) together with well-known arguments imply the existence of $A_{2}>0$ and $B_{2}>0$ such that

$$
|f(z)| \leq A_{2} \exp \left(R|\operatorname{Im} z|+B_{2} \omega(z)\right), \quad z \in \mathbb{C} .
$$

This shows that $f \in A_{(\omega, R)}$. By the definition of $f$ and $\lambda$ we have

$$
\varrho(f)=\left(\varrho_{j}\left(\left.f\right|_{S_{j}}\right)\right)_{j \in \mathbb{N}}=\left(\varrho_{j}\left(\lambda_{j}\right)\right)_{j \in \mathbb{N}}=y .
$$

Therefore, $\varrho: A_{(\omega, R)} \rightarrow K\left(\alpha, \beta,\left(E_{j}\right)_{j \in \mathbb{N}}\right)$ is surjective. Thus we have proved that $A_{(\omega, R)} / M_{F} A_{(\omega, R)} \cong K_{\infty}\left(\alpha, \beta,\left(E_{j}\right)_{j \in \mathbb{N}}\right)$ by the open mapping theorem. 
Since $A_{(\omega, R)}$ is a (DFN)-space by Lemma $4.5(\mathrm{a})$, also $K_{\infty}\left(\alpha, \beta,\left(E_{j}\right)_{j \in \mathbb{N}}\right)$ is a (DFN)-space. It is easy to check that

$$
D: K_{\infty}\left(\alpha, \beta,\left(E_{j}\right)_{j \in \mathbb{N}}\right) \rightarrow K_{\infty}\left(\alpha,\left(E_{j}\right)_{j \in \mathbb{N}}\right), \quad D\left(\left(x_{j}\right)_{j \in \mathbb{N}}\right):=\left(e^{-\beta_{j}} x_{j}\right)_{j \in \mathbb{N}}
$$

is a linear topological isomorphism. Hence $K_{\infty}\left(\alpha,\left(E_{j}\right)_{j \in \mathbb{N}}\right)$ is a (DFN)-space. By Meise [15, Propositions 1.3 and 1.4], this implies that $K_{\infty}\left(\alpha,\left(E_{j}\right)_{j \in \mathbb{N}}\right)$ and hence also $K_{\infty}\left(\alpha, \beta,\left(E_{j}\right)_{j \in \mathbb{N}}\right)$ is isomorphic to the strong dual of a nuclear power series space of infinite type.

4.8. Lemma. Let $\omega$ be a (DN)-weight function or a strong weight function. If $F \in A_{(\omega)}$ is $(\omega)$-slowly decreasing then for each $R>0$ the map $M_{F}: A_{(\omega, R)} \rightarrow A_{(\omega, R)}$ admits a continuous linear left inverse.

Proof. By Lemma 4.6, the following sequence is exact:

$$
0 \rightarrow A_{(\omega, R)} \stackrel{M_{F}}{\longrightarrow} A_{(\omega, R)} \stackrel{q}{\rightarrow} A_{(\omega, R)} / M_{F}\left(A_{(\omega, R)}\right) \rightarrow 0,
$$

where $q$ denotes the quotient map. Since the maps $M_{F}$ and $q$ are linear and continuous, it follows from Meise and Vogt [22, Proposition 26.4] that the dual sequence

$$
0 \rightarrow\left(A_{(\omega, R)} / M_{F}\left(A_{(\omega ; R)}\right)\right)^{\prime} \stackrel{q^{\prime}}{\rightarrow} A_{(\omega, R)}^{\prime} \stackrel{M_{F}^{\prime}}{\longrightarrow} A_{(\omega, R)}^{\prime} \rightarrow 0
$$

is exact as well. By Lemma 4.5, the space $A_{(\omega, R)}^{\prime}$ has (DN), while by Lemma 4.7, the space $\left(A_{(\omega, R)} / M_{F}\left(A_{(\omega, R)}\right)\right)^{\prime}$ has $(\Omega)$. Hence the exact sequence (4.19) splits by the splitting theorem of Vogt and Wagner (see [22, 30.1]), which finishes the proof.

4.9. Proposition. Let $\omega$ be a (DN)-weight function or a strong weight function. Assume that for $\mu \in \mathcal{E}_{(\omega)}^{\prime}(\mathbb{R})$ its Fourier-Laplace transform $\widehat{\mu}$ is $(\omega)$-slowly decreasing and is in $A_{(\omega)}$. Then for each $a, b \in \mathbb{R}$ with $a<b$ the sequence

$$
0 \rightarrow \operatorname{ker} T_{\mu,[a, b]} \rightarrow \mathcal{E}_{(\omega)}[a, b] \stackrel{T_{\mu,[a, b]}}{\longrightarrow} \mathcal{E}_{(\omega)}[a, b] \rightarrow 0
$$

is exact and splits.

Proof. From Lemma 4.5 we know that for each $R>0$ the space $\mathcal{E}_{(\omega)}^{\prime}[-R, R]$ is isomorphic to $A_{(\omega, R)}$ via the Fourier-Laplace transform. From this and the fact that $T_{\mu}$ commutes with translations it follows that for $R:=(a+b) / 2$ and up to isomorphism the present sequence is identical with the exact sequence (4.19). Hence it is exact and splits.

Remark. If we replace in Proposition 4.9 the condition " $\widehat{\mu}$ is $(\omega)$-slowly decreasing" by " $T_{\mu,[a, b]}$ is surjective for some interval $[a, b]$ ", then it follows as in the proof of Proposition 4.9 that $T_{\mu,[a, b]}: \mathcal{E}_{(\omega)}[a, b] \rightarrow \mathcal{E}_{(\omega)}[a, b]$ admits a continuous linear right inverse. 
REMARK. If $\omega$ is a strong weight function then the existence of a continuous linear right inverse for $T_{\mu}$ as in Proposition 4.9 can also be proved in the following way. By Meise and Taylor [20, Theorem 3.1], there exists a continuous linear extension operator $E_{0}: \mathcal{E}_{(\omega)}[a, b] \rightarrow \mathcal{E}_{(\omega)}(\mathbb{R})$. If we fix $\varphi \in \mathcal{D}_{(\omega)}(\mathbb{R})$ such that $\varphi \equiv 1$ in some neighborhood of $[a, b]$, then $E: f \mapsto \varphi E_{0}(f)$ defines a continuous linear operator from $\mathcal{E}_{(\omega)}[a, b]$ into $\mathcal{D}_{(\omega)}(\mathbb{R})$. Since $T_{\mu}$ admits a fundamental solution $\nu \in \mathcal{D}_{(\omega)}^{\prime}(\mathbb{R})$ it is easy to check that the map

$$
R: \mathcal{E}_{(\omega)}[a, b] \rightarrow \mathcal{E}_{(\omega)}[a, b], \quad R(f):=\left.\nu *(E(f))\right|_{[a, b]},
$$

is a continuous linear right inverse for $T_{\mu}$.

4.10. EXAMPLE. Let $\omega$ be a quasianalytic (DN)-weight function which satisfies condition $\left(\alpha_{1}\right)$. Then there exist $(\omega)$-ultradifferential operators $T_{\mu}$ which admit a continuous linear right inverse on $\mathcal{E}_{(\omega)}[a, b]$, but which do not admit a continuous linear right inverse on $\mathcal{E}_{(\omega)}(\mathbb{R})$.

To show this, let $\left(b_{j}\right)_{j \in \mathbb{N}}$ be an increasing sequence in $] 0, \infty[$ which satisfies, for $n(t):=\operatorname{card}\left\{j \in \mathbb{N}: b_{j} \leq t\right\}$,

(i) $b_{j+1} \geq 4 b_{j}, j \in \mathbb{N}$,

(ii) $n(t) \log t=o(\omega(t))$ as $t \rightarrow \infty$.

Then define

$$
F(z):=\prod_{j \in \mathbb{N}}\left(1-\frac{z}{i b_{j}}\right), \quad z \in \mathbb{C} .
$$

By Rudin [28, Theorem 15.6], $F$ is an entire function. Arguing as in [6, Lemma 3.5] we get the existence of $B>0$ such that

$$
|F(z)| \leq B \exp (B \omega(z)), \quad z \in \mathbb{C},
$$

and the existence of $\varepsilon_{0}>0$ and $C_{0}>0$ such that for each $w \in \mathbb{C} \backslash$ $\bigcup_{j \in \mathbb{N}} B\left(i b_{j}, 1\right)$ we have

$$
|F(w)| \geq \varepsilon_{0} \exp \left(-C_{0} \omega(w)\right) .
$$

Therefore, $F$ is in $A_{(\omega)}$ and it follows from Lemma 4.6 that $F$ is $(\omega)$-slowly decreasing. By Theorem 2.6 we can find $\mu \in \mathcal{E}_{(\omega)}^{\prime}(\mathbb{R})$ such that $\widehat{\mu}=F$. It then follows from Proposition 4.9 that $T_{\mu}$ admits a continuous linear right inverse on $\mathcal{E}_{(\omega)}[a, b]$ for each $a, b \in \mathbb{R}$ with $a<b$. However, since condition $3.2(\mathrm{~b})$ does not hold for $F$, it follows from Proposition 3.2 that $T_{\mu}: \mathcal{E}_{(\omega)}(\mathbb{R}) \rightarrow \mathcal{E}_{(\omega)}(\mathbb{R})$ does not admit a continuous linear right inverse.

From Theorem 3.4 we now get the following corollary.

4.11. COROLlary. Let $\omega$ be a quasianalytic $(\mathrm{DN})$-weight function which satisfies condition $\left(\alpha_{1}\right)$. Assume that for $\mu \in \mathcal{E}_{(\omega)}^{\prime}(\mathbb{R})$ its Fourier-Laplace transform $\widehat{\mu}$ is $(\omega)$-slowly decreasing and is in $A_{(\omega)}$. Then the following assertions are equivalent: 
(1) $T_{\mu}: \mathcal{E}_{(\omega)}(\mathbb{R}) \rightarrow \mathcal{E}_{(\omega)}(\mathbb{R})$ admits a continuous linear right inverse.

(2) There exists $C>0$ such that $|\operatorname{Im} a| \leq C(\omega(a)+1)$ for each $a \in V(\widehat{\mu})$.

(3) For each/some $a, b \in \mathbb{R}$ with $a<b$ and each $f \in \operatorname{ker} T_{\mu,[a, b]}$ there exists $g \in \operatorname{ker} T_{\mu}$ such that $f=\left.g\right|_{[a, b]}$.

Proof. (1) $\Rightarrow(2)$. This holds by Proposition 3.2.

$(2) \Rightarrow(3)$. Fix any $R>0$ and consider the following commutative diagram with exact rows:

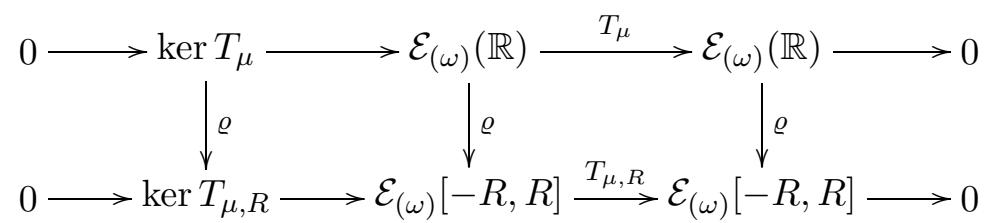

where the vertical maps $\varrho$ are defined by $\varrho(f):=\left.f\right|_{[-R, R]}$. They are linear and continuous. Therefore, the dual diagram is commutative as well. If we apply the Fourier-Laplace transform to it, then we get the following commutative diagram with exact rows:

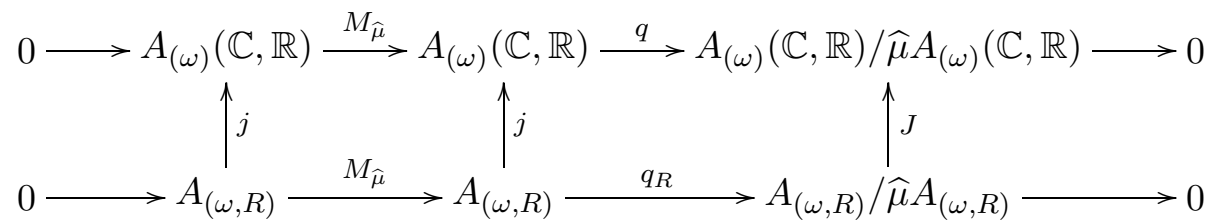

where the maps $j$ are obvious inclusions and where $J$ is induced by $j$. As we proved in Proposition 3.3 the quotient $A_{(\omega)}(\mathbb{C}, \mathbb{R}) / \widehat{\mu} A_{(\omega)}(\mathbb{C}, \mathbb{R})$ is isomorphic to $K_{\infty}\left(\gamma,\left(E_{j}\right)_{j \in \mathbb{N}}\right)$. Since condition (2) holds, we also know from the proof of this proposition that there exists $A_{2}>0$ such that for each component $S$ of $S\left(\widehat{\mu}, \varepsilon_{0}, C_{0}\right)$ which satisfies $S \cap V(\widehat{\mu}) \neq 0$ we have $|\operatorname{Im} z| \leq A_{2} \omega(z)+A_{2}$ for each $z \in S$. This implies that the space $K_{\infty}\left(\lambda,\left(E_{j}\right)_{j \in \mathbb{N}}\right)$ is identical with $K_{\infty}\left(\alpha, \beta,\left(E_{j}\right)_{j \in \mathbb{N}}\right)$ defined in the proof of Lemma 4.7. Moreover, the map $J$ in the diagram above is the identity if we identify both spaces. Hence $J$ and consequently $\varrho: \operatorname{ker} T_{\mu} \rightarrow \operatorname{ker} T_{\mu, R}$ is an isomorphism. Since $T_{\mu}$ commutes with translations, this implies (3) for each $a<b$ in $\mathbb{R}$.

$(3) \Rightarrow(1)$. Assume that condition (3) holds for some $a, b \in \mathbb{R}$. Then let $R:=(a+b) / 2$ and note that the present hypothesis and Proposition 4.9 imply that $T_{\mu, R}: \mathcal{E}_{(\omega)}[-R, R] \rightarrow \mathcal{E}_{(\omega)}[-R, R]$ admits a continuous linear right inverse $S_{R}$. To use it in order to define a continuous linear right inverse for $T_{\mu}$, we fix any $g \in \mathcal{E}_{(\omega)}(\mathbb{R})$. Since $T_{\mu}$ is surjective by hypothesis, there exists $f \in \mathcal{E}_{(\omega)}(\mathbb{R})$ with $T_{\mu} f=g$. We denote by $\varrho: \mathcal{E}_{(\omega)}(\mathbb{R}) \rightarrow \mathcal{E}_{(\omega)}[-R, R]$ the restriction map $\varrho(h):=\left.h\right|_{[-R, R]}$ and we let

$$
h_{R}:=\varrho(f)-S_{R}(\varrho(g)) .
$$


Then $h_{R}$ belongs to $\mathcal{E}_{(\omega)}[-R, R]$ and

$T_{\mu, R}\left(h_{R}\right)=T_{\mu, R}(\varrho(f))-T_{\mu, R} \circ S_{R}(\varrho(g))=\varrho\left(T_{\mu}(f)\right)-\varrho(g)=\varrho(g)-\varrho(g)=0$

implies $h_{R} \in \operatorname{ker} T_{\mu, R}$. By the present hypothesis we can choose $H \in \operatorname{ker} T_{\mu}$ satisfying $\varrho(H)=h_{R}$. Now we let

$$
S(g):=f-H
$$

and claim that $S(g)$ is well-defined, i.e., that it does not depend on the choice of $f$. To show this, assume that $f_{1} \in \mathcal{E}_{(\omega)}(\mathbb{R})$ satisfies $T_{\mu} f_{1}=g$. Then $h_{1, R}:=\varrho\left(f_{1}\right)-S_{R}(\varrho(g))$ is again in $\operatorname{ker} T_{\mu, R}$ and we can choose $H_{1} \in \operatorname{ker} T_{\mu}$ satisfying $\varrho\left(H_{1}\right)=h_{1, R}$. Now note that our choices imply

$$
\begin{aligned}
\varrho(f-H) & =\varrho(f)-\varrho(H)=\varrho(f)-h_{R}=S_{R}(\varrho(g)), \\
\varrho\left(f_{1}-H_{1}\right) & =\varrho\left(f_{1}\right)-\varrho\left(H_{1}\right)=\varrho\left(f_{1}\right)-h_{1, R}=S_{R}(\varrho(g)) .
\end{aligned}
$$

Hence $f-H$ and $f_{1}-H_{1}$ coincide on the interval $[-R, R]$. Since $\omega$ is a quasianalytic weight function, this implies $f-H=f_{1}-H_{1}$ and proves that $S(g)$ is well-defined. From this it follows easily that $S: \mathcal{E}_{(\omega)}(\mathbb{R}) \rightarrow \mathcal{E}_{(\omega)}(\mathbb{R})$ is a linear map. By the definition of $S$ we have

$$
\varrho(S(g))=\varrho(f)-\varrho(H)=\varrho(f)-h_{R}=S_{R}(\varrho(g)) .
$$

In order to show that $S$ is continuous, we apply the closed graph theorem. To do so, let $\left(g_{j}\right)_{j \in \mathbb{N}}$ be any sequence in $\mathcal{E}_{(\omega)}(\mathbb{R})$ which satisfies $g_{j} \rightarrow g$ and $S\left(g_{j}\right) \rightarrow f$ in $\mathcal{E}_{(\omega)}(\mathbb{R})$. Then the equality $\varrho\left(S\left(g_{j}\right)\right)=S_{R}\left(\varrho\left(g_{j}\right)\right), j \in \mathbb{N}$, implies that the sequence $\left(\varrho\left(S\left(g_{j}\right)\right)\right)_{j \in \mathbb{N}}$ converges to $\varrho(f)$ as well as to $S_{R}(\varrho(g))=$ $\varrho(S(g))$. Hence $f$ and $S(g)$ coincide on $[-R, R]$ and consequently $f=S(g)$. Thus $S$ has a closed graph and therefore is continuous. Since

$$
T_{\mu}(S(g))=T_{\mu}(f-H)=T_{\mu}(f)-T_{\mu}(H)=T_{\mu}(f)=g
$$

for each $g \in \mathcal{E}_{(\omega)}(\mathbb{R})$, we proved (1).

\section{References}

[1] C. A. Berenstein and R. Gay, Complex Analysis and Special Topics in Harmonic Analysis, Springer, New York, 1995.

[2] C. A. Berenstein and B. A. Taylor, A new look at interpolation theory for entire functions of one variable, Adv. Math. 33 (1979), 109-143.

[3] -, - Interpolation problems in $\mathbb{C}^{n}$ with application to harmonic analysis, J. Anal. Math. 38 (1980), 188-259.

[4] J. Bonet and P. Domański, Parameter dependence of solutions of differential equations on spaces of distributions and the splitting of short exact sequences, J. Funct. Anal. 230 (2006), 329-381.

[5] J. Bonet, A. Galbis and R. Meise, On the range of convolution operators on nonquasianalytic ultradifferentiable functions, Studia Math. 126 (1997), 171-198.

[6] J. Bonet and R. Meise, Quasianalytic functionals and projective descriptions, Math. Scand. 94 (2004), 249-266. 
[7] R. W. Braun, R. Meise and B. A. Taylor, Ultradifferentiable functions and Fourier analysis, Result. Math. 17 (1990), 206-237.

[8] R. W. Braun, R. Meise and D. Vogt, Existence of fundamental solutions and surjectivity of convolution operators on classes of ultradifferentiable functions, Proc. London Math. Soc. 61 (1990), 344-370.

[9] P. Domański and D. Vogt, Linear topological properties of the space of analytic functions on the real line, in: Recent Progress in Functional Analysis, K.-D. Bierstedt et al. (eds.), North-Holland Math. Stud. 189, North-Holland, Amsterdam, 2001, $113-132$.

[10] T. Heinrich and R. Meise, A support theorem for quasianalytic functionals, Math. Nachr. 280 (2007), 364-387.

[11] L. Hörmander, On the range of convolution operators, Ann. of Math. 76 (1962), 148-170.

[12] - An Introduction to Complex Analysis in Several Variables, Van Nostrand, Princeton, NJ, 1966.

[13] M. Langenbruch, Continuous linear right inverses for convolution operators in spaces of real analytic functions, Studia Math. 110 (1994), 65-82.

[14] B. Ya. Levin, Distribution of Zeros of Entire Functions, Transl. Math. Monogr. 5, Amer. Math. Soc., 1980.

[15] R. Meise, Sequence space representations for (DFN)-algebras of entire functions modulo closed ideals, J. Reine Angew. Math. 363 (1985), 59-95.

[16] - Structure of closed linear translation invariant subspaces of $A(\mathbb{C})$ and kernels of analytic convolution operators, in: Functional Analysis: Surveys and Recent Results III, K.-D. Bierstedt and B. Fuchssteiner (eds.), North-Holland Math. Stud. 90, North-Holland, Amsterdam, 1984, 331-347.

[17] R. Meise and B. A. Taylor, Whitney's extension theorem for ultradifferentiable functions of Beurling type, Ark. Mat. 26 (1988), 265-287.

[18] - - - Splitting of closed ideals in (DFN)-algebras of entire functions and the property (DN), Trans. Amer. Math. Soc. 302 (1987), 341-370.

[19] - - - A decomposition lemma for entire functions and its applications to spaces of ultradifferentiable functions, Math. Nachr. 142 (1989), 45-72.

[20] - - - Linear extension operators for ultradifferentiable functions of Beurling type on compact sets, Amer. J. Math. 111 (1989), 309-337.

[21] R. Meise and D. Vogt, Characterization of convolution operators on spaces of $C^{\infty}$ functions admitting a continuous linear right inverse, Math. Ann. 279 (1987), 141155.

[22] - - - Introduction to Functional Analysis, Oxford Univ. Press, Oxford, 1997.

[23] T. Meyer, Die Fourier-Laplace Transformation quasianalytischer Funktionale und ihre Anwendung auf Faltungsoperatoren, Diplomarbeit, Düsseldorf, 1989.

[24] —, Surjectivity of convolution operators on spaces of ultradifferentiable functions of Roumieu type, Studia Math. 125 (1997), 101-129.

[25] S. Momm, Closed principal ideals in nonradial Hörmander algebras, Arch. Math. (Basel) 58 (1992), 47-55.

[26] H. J. Petzsche and D. Vogt, Almost analytic extension of ultradifferentiable functions and the boundary values of holomorphic functions, Math. Ann. 267 (1984), 17-35.

[27] T. Rösner, Surjektivität partieller Differentialoperatoren auf quasianalytischen Roumieu-Klassen, Dissertation, Düsseldorf, 1997.

[28] W. Rudin, Real and Complex Analysis, 2nd ed., McGraw-Hill, New York, 1974. 
[29] B. A. Taylor, Linear extension operators for entire functions, Michigan Math. J. 29 (1982), 185-197.

[30] D. Vogt, Topological properties of spaces $H^{\omega}$, preprint, 2002.

IMPA-UPV and Dpto. de Matemática Aplicada

Universidad Politécnica de Valencia

E-46071 Valencia, Spain

E-mail: jbonet@mat.upv.es
Mathematisches Institut Heinrich-Heine-Universität Universitätsstrasse 1 40225 Düsseldorf, Germany E-mail: meise@math.uni-duesseldorf.de

Received April 5, 2007

Revised version September 27, 2007 